\title{
Stretch-induced endogenous electric fields drive neural crest directed collective cell migration in vivo
}

Fernando Ferreira $^{1}$, Sofia Moreira ${ }^{1}$ and Elias H. Barriga ${ }^{1 *}$

${ }^{1}$ Mechanisms of Morphogenesis Lab, Gulbenkian Institute of Science (IGC), Oeiras, Portugal.

* Correspondence to ebarriga@igc.gulbenkian.pt

Keywords: Endogenous electric fields, stretch-activated channels, directed collective cell migration, neural crest cells, Xenopus laevis. 


\begin{abstract}
Directed collective cell migration (dCCM) is essential for morphogenesis ${ }^{1,2}$. Cell clusters migrate in inherently complex in vivo environments composed of chemical, electrical, mechanical as well as topological features. While these environmental factors have been shown to allow dCCM in vitro, our understanding of $\mathrm{dCCM}$ in vivo is mostly limited to chemical guidance $^{3}$. Thus, despite its wide biological relevance, the mechanisms that guide dCCM in vivo remain unclear. To address this, we study endogenous electric fields in relation to the migratory environment of the Xenopus laevis cephalic neural crest, an embryonic cell population that collectively and directionally migrates in vivo ${ }^{4}$, and whose migratory mode has been linked to cancer invasion and metastasis ${ }^{5}$. Combining bioelectrical, biomechanical and molecular tools, we show that endogenous electric fields drive neural crest dCCM via electrotaxis in vivo. Moreover, we identify the voltage-sensitive phosphatase 1 (Vsp1) as a key component of the molecular mechanism used by neural crest cells to transduce electric fields into a directional cue in vivo. Furthermore, Vsp1 function is specifically required for electrotaxis, being dispensable for cell motility and chemotaxis. Finally, we reveal that endogenous electric fields are mechanoelectrically established. Mechanistically, convergent extension movements of the neural fold generate membrane tension, which in turn opens stretch-activated channels to mobilise the ions required to fuel electric fields. Overall, our results reveal a mechanism of cell guidance, where electrotaxis emerges from the mechanoelectrical and molecular interplay between neighbouring tissues. More broadly, our data contribute to validate the, otherwise understudied, functions of endogenous bioelectrical stimuli in morphogenetic processes ${ }^{6}$.
\end{abstract}




\section{Main}

Cephalic neural crest cells are induced at the interface between the neural plate and the non-neural ectoderm, from where they migrate following stereotypical mediolateral paths known as streams ${ }^{7}$ (Fig. 1a). The migration of neural crest cells has been widely studied; yet, how do they attain directionality in convoluted native contexts remains unclear. Chemical cues have been proposed to guide the directed collective cell migration (dCCM) of neural crest cells via chemotaxis ${ }^{8}$. However, recent evidence suggested that chemotaxis may not be sufficient to explain their directed motion in vivo ${ }^{9}$. Intriguingly, in vitro experiments have demonstrated the ability of neural crest cells to directionally migrate by following extrinsically imposed electric fields, in a process termed as electrotaxis (or galvanotaxis) ${ }^{10-12}$. In addition, seminal work detected the presence of electric fields in amphibian embryos during neurulation ${ }^{13,14}$ and the application of external electric fields led to developmental defects ${ }^{15}$. In this scenario, electrotaxis emerges as an alternative or complementary mechanism to explain neural crest dCCM in vivo. Nonetheless, whether and how endogenous electric fields drive directed cell migration via electrotaxis in native contexts remains unclear.

To address this, we first used ultrasensitive vibrating probes to map extracellular electric currents ${ }^{16}$ along the migratory path of neural crest cells. We performed our measurements from the neural fold towards the flanking non-neural ectoderm and from non-migratory (stage 13) to migratory (stage 22) stages of neural crest cells (Fig. 1a; Supplementary Fig.. 1). Our spatiotemporal measurements detected a long-lasting and sustained dipole loop, with outward currents registered in the neural fold and inward currents in the flanking non-neural ectoderm (Fig. 1b). Thus, based in Ohm's law ${ }^{17}$ (Methods), this pattern of electric currents indicates that a mediolateral endogenous electric field emerges in the neural crest migratory path from early stages of migration, with the cathode (- pole) in the neural fold and the anode (+ pole) in the flanking non-neural ectoderm (Fig. 1c). Furthermore, we observed that outward currents reached a maximum at stages in which neural crest cells are preparing to migrate (pre-migratory stages 17 and 20) and that these values decreased once migration started (Fig. 1b). Despite these differences in the absolute magnitude of current, the slope of the electric field established in the migratory path of neural crest cells remains unaltered from pre-migratory stages (Fig. 1d). These results indicate that neural crest cells may experience electric fields towards the onset of their dCCM.

To dissect the role of these endogenous electric fields in neural crest directed cell migration, we generated an ex vivo electrotaxis assay capable of reproducing the recorded 
endogenous electric fields in a controlled microenvironment (Fig. 1e; Methods). In the absence of an electric field, cultured neural crests clusters tend to radially disperse and to perform random migration (Supplementary Video 1), but when an electric field was applied, the collectiveness of these clusters is retained and the groups of neural crest cells persistently and collectively migrated towards the anode (Fig. 1f,g; Supplementary Video 1). Interestingly, switching the polarity of the electric field led to a switch in the direction of migration towards the new anodal position (Fig. 1h; Supplementary Video 1). Note that this direction of migration agrees with the orientation of the endogenous electric field recorded in vivo (Fig. 1d). These observations were quantitatively confirmed by computing the forward migration index (FMI) as a readout of the directionality displayed by clusters in each condition (Fig. 1i); while FMIs closer to 0 represent random migration, FMIs near 1 or -1 account for a directional response (Methods). Since the recorded endogenous electric fields ranged from $\sim 10-100 \mathrm{mV}$ $\mathrm{mm}^{-1}$, we tested the response of neural crest cells to electric fields of 10,25 and $100 \mathrm{mV} \mathrm{mm} \mathrm{m}^{-1}$ (Methods). Our assays confirmed that the directionality of neural crest cells was consistent in the range of electric fields analysed and that, until some extent, cluster directionality depends on the level of the applied electric fields (Supplementary Fig.. 2; Supplementary Video 2). Next, we addressed whether the directional response of neural crest cells to electric fields is an emergent property of the collective or also appears in individual cells. By comparing the response of neural crest clusters with the behaviour of single cells exposed to the same electric field, we found that isolated cells displayed FMIs closer to 0, whereas clusters displayed effective anodal response (Supplementary Fig.. 3; Supplementary Video 3). Together, these results indicate that physiological levels of electric fields influence the directionality of neural crest cells and consequently their collective migration ex vivo. Thus, to confirm that these electric fields are relevant for neural crest migration in vivo, we perturbed the endogenous electric fields by extrinsically applying antiparallel electric fields to the neural crest migratory path (Fig. 1j; Methods). While control neural crests migrated by following stereotypical streams (a hallmark of $\mathrm{dCCM}^{18,19}$ ), the antiparallel electric field consistently impaired neural crest streams formation, as shown by in situ hybridisation of a migratory neural crest marker (Fig. 1k,l). Altogether, our data indicate that endogenous electric fields are required for the $\mathrm{dCCM}$ of neural crest cells in vivo, introducing electrotaxis as a complementary or alternative mechanism by which the neural crest attain their characteristic directionality.

To explore this possibility and to gain insights into the mechanism of electrotaxis in vivo, we investigated how neural crest cells sense and transduce endogenous electric fields into 
a directional cue. Most of the proteins reported to mediate electrotaxis are part of the cell selfpolarity ${ }^{20,21}$ or cell-substrate adhesion ${ }^{22,23}$ machineries and few molecules have been shown to specifically mediate electrotaxis in vitro ${ }^{24-27}$. Hence, the precise mechanism used by cells to transduce electric fields into a directional response in vivo remains unclear. To address this point, we searched for candidate molecules for the mediation of electrotaxis (electrosensors) in the neural crest by performing RNA-seq experiments from isolated neural crest clusters (Supplementary Fig.. 4). Among other candidates, we found that the voltage-sensitive phosphatase 1 (originally described as tpte2.L and referred here as $v s p 1)^{28,29}$ was enriched in our neural crest-specific library, whereas the paralogous gene vsp2 (tpte2.S) was not expressed (Supplementary Table. 1; Supplementary Fig.. 4). Vsp1 contains a channel-like transmembrane domain that senses voltage fluctuations and a cytosolic catalytic domain with phosphatase activity ${ }^{28,30}$. This peculiar protein structure positioned Vsp1 as a good candidate to sense and transduce electrical stimuli into a cascade that could eventually lead to electrotaxis. To test this, we first validated the expression of Vsp1 in neural crest cells and confirmed its localization to the cell membrane, where it is expected to operate upon membrane depolarization (Supplementary Fig.. 4). Then, we functionally tested the requirement of Vsp1 activity for neural crest electrotaxis ex vivo, by expressing a catalytically inactive form of Vsp1 (Vsp1-C301S) previously shown to reduce the phosphatase activity in Xenopus oocytes to applied voltage ${ }^{29,31}$. In the absence of electric fields, Vsp1-C301S injected neural crest cells did not display any significant effects in motility, as observed in our ex vivo assays (Supplementary Fig.. 5; Supplementary Video 4). However, when an electric field was applied, Vsp1-C301S injected clusters failed to directionally respond, unlike control clusters that effectively performed electrotaxis (Fig. 2a-c; Supplementary Video 5), strongly suggesting a role for Vsp1 in collective electrotaxis. To further test the involvement of Vsp1 in electrotaxis, we investigated whether Vsp1 activity is sufficient to allow directed migration at suboptimal levels of electric fields. First, we determined that electrotaxis of wild-type neural crest clusters was minimal when exposed to electric fields of $5 \mathrm{mV} \mathrm{mm}^{-1}$ ex vivo (Fig. 2d). Then, we injected a mutant form of Vsp1 which is more sensitive to voltage (VSP1-R152QGFP) $)^{29,31}$ and analysed its impact in neural crest electrotaxis. VSP1-R152Q-GFP expression was sufficient to significantly increase the frequency of neural crest clusters displaying a persistent and directional response under these suboptimal electric fields (Fig. 2d-f; Supplementary Video 6), confirming that Vsp1 activity modulates the sensitivity of cells to electric fields. 
Next, we tested the requirement of Vsp1 for electrotaxis in vivo by analysing neural crest migration in embryos injected with Vsp1-C301S. Vsp1 loss-of-function showed a consistent decrease in the formation of stereotypical streams, as shown by in situ hybridisation analyses (Supplementary Fig.. 5), suggesting an effect in directionality. Hence, we next performed graft experiments in which control or Vsp1-C301S nuclei-tagged neural crest cells were transplanted into wild-type untagged embryos. Our analyses of cell trajectories revealed that while control cells effectively migrated by forming stereotypical streams, the directionality of Vsp1-C301S injected cells was significantly reduced in vivo (Fig. 2g-j; Supplementary Video 7). Furthermore, since chemotaxis has been also proposed to guide neural crest migration $^{32}$, we exposed control and Vsp1-C301S injected clusters to a previously described chemotaxis assay relying in the response of neural crest clusters to the chemokine stromal cellderived factor 1 (SDF-1, also known as CXCL12) ${ }^{32}$. Our results showed that Vsp1-C301S injection did not affect the neural crest response towards SDF-1 coated beads (Supplementary Fig.. 6; Supplementary Video 8). Together, these data sets indicate that, while not being required for neural crest motility or chemotaxis, Vsp1 is essential for the response of the neural crest to electric fields and in turn for collective electrotaxis in vivo. Since the role of Vsp1 in electrotaxis has not been previously described, we predict that its study can have major implications across research fields. Indeed, by accessing publicly available databases, we found that orthologous VSP is expressed in several mammalian cell types, which are used as wound healing and cancer models and that have been shown to electrotax at least in vitro (Supplementary Table. 2), suggesting a requirement of Vsp1 in these biological contexts.

Our next goal was to explore the mechanism underlying the emergence of the endogenous electric fields ${ }^{33}$. Previous work has shown that membrane stretching activates ion channels to allow for ion translocation ${ }^{34}$. In addition, stress measurements in amphibians detected an increase of anisotropic tension in the neural plate, but isotropic tension in the nonneural ectoderm ${ }^{35-37}$. Further work showed that this anisotropic tension arises in the neural plate owing to the activity of the planar cell polarity (PCP) pathway in promoting convergent extension movements of the ectoderm ${ }^{38,39}$. Thus, we hypothesised that the outward currents recorded in the neural fold, and in turn electric fields, emerge from ionic movement due to the stretching of neural fold cell membranes, owing to PCP-mediated convergent extension movements within the ectoderm. To test this hypothesis, we first confirmed that there was a tension build-up in the membrane of neural fold cells from stages in which outward electric currents were minimal or undetected (stage 13) to stages in which we recorded high levels of 
outward electric currents (stage 17), using laser ablation experiments (Fig. 3a,b; Supplementary Fig.. 8; Supplementary Video 9). As this increased tension correlated with the emergence of electric fields, we next analysed the impact of PCP inhibition in neural fold to membrane tension and electric field formation (Fig. 3a). PCP was inhibited by targeted injections of DshDEP ${ }^{+}$, a known PCP inhibitor ${ }^{39}$, into the neural fold (Supplementary Fig.. 7). $\mathrm{DshDEP}^{+}$injection led to a significant decrease in neural fold membrane tension (Fig. 3b; Supplementary Fig.. 8; Supplementary Video 9), as well as to a drastic reduction on the neural fold outward currents that, in turn, affected the establishment of endogenous electric fields (Fig. 3c,d; Supplementary Fig.. 8). Thus, we next tested whether the activity of stretchactivated channels was required for the formation of electric fields. For this, we performed livecurrent recordings before, during and after GsMTx4 inoculation, a peptide toxin which specifically inhibits stretch-activated channels in Xenopus and in other species ${ }^{40,41}$ (Fig. 3e). We observed that shortly after GsMTx4 inoculation, the outward currents significantly decreased, abolishing the establishment of endogenous electric fields when comparing to the control (Fig. 3f). Furthermore, we found that neural crest cells dCCM in vivo was also impacted by the inhibition of PCP in the neural fold (Supplementary Fig.. 7), alike to the phenotype observed upon Vsp1 loss-of-function (Supplementary Fig.. 5). Importantly, this impact in migration is non-cell autonomous, since wild-type neural crest cells fail to directionally migrate once grafted into a host expressing $\mathrm{DshDEP}^{+}$in the neural fold (Fig. 3g-j; Supplementary Video 10). Combined, our experiments show that PCP-mediated neural fold cell membrane stretching is required to the emergence of electric fields in the embryo and, consequently, to the directed migration of neural crest cells.

Finally, to confirm that the phenotype in the neural crest $\mathrm{dCCM}$ is a consequence of the effect of PCP in electric field formation, we assessed whether the introduction of a parallel exogenous electric field of identical magnitude and direction to the ones recorded in vivo would rescue PCP associated defects (Fig. 4a,c). In the absence of a parallel electric field, PCP inhibition in the neural fold non-autonomously inhibited neural crest dCCM in vivo (Fig. 4a,b,e,f), as we previously observed (Fig. 3f,g). Remarkably, the introduction of the parallel electric field rescued the dCCM of wild-type neural crests in these embryos (Fig. 4c-f). This electric field-induced rescue was lost when Vsp1-C301S was injected in the neural crest, further confirming the requirement of Vsp1 in the response to electric fields (Fig. $\mathbf{4 c - f}$ ). These results confirm the role of the recorded electric fields in guiding neural crest $\mathrm{dCCM}$ via electrotaxis in 
vivo and the requirement of Vsp1 as an important part of the electrosensitive cascade by which neural crest cells transduce these endogenous electric fields into dCCM.

A large body of in vitro evidence supports the idea that electrotaxis directs cell migration $^{17,24}$. In vivo, some hypotheses have been proposed to explain the establishment of endogenous electric fields ${ }^{33}$, although no experimental demonstration was provided to date. Here, we unveiled that endogenous electric fields are established in the migratory path of neural crest cells by PCP-driven mechanical stretching of the neural fold cell membranes. Furthermore, we demonstrated that the specific activity of Vsp1 in neural crest cells converts these endogenous electric fields into collective electrotaxis, which agrees with the topology of the electric fields recorded in the migratory path of neural crest cells in vivo (Fig. 4g). Thus, our research contributes to advance the accelerating field of electrotransduction ${ }^{24,25,42}$ by positioning Vsp1 as an electrosensor/transducer that has the potential to operate in several biological contexts (Supplementary Table. 2). More broadly, our work emphasises the idea that the occurrence of morphogenetic events such as dCCM are synchronised by the interplay between surrounding tissues, not only at the molecular, but also at the electrical and mechanical levels.

Thus, given the wide relevance of bioelectricity and directed cell migration in a variety of biological processes such as embryogenesis, tissue repair and cancer ${ }^{1,6,16,43,44}$, we believe that our results can deeply impact the research across these fields. Moreover, our research has the potential to influence the growing field of bioartificial organ production ${ }^{45}$, as this field may benefit from the inclusion of endogenous electric fields and from considering mechanoelectrical interplay in their protocols. 


\section{Acknowledgments}

The authors would like to thank Dr Benjamin Steventon (Cambridge University), Dr Ana Patrícia Ramos (IGC) and Dr Eric Theveneau (CBI-Toulouse) for helpful comments to the manuscript. In addition, the authors thank all lab members for helpful discussion, João Mata for technical assistance and Cristian Marchant for RNA extraction; Eric Theveneau for plasmids and in situ hybridization probes; and the IGC's Advanced imaging (supported by PPBI-POCI-01-0145-FEDER-022122), Genomics, Bioinformatics and Aquatic animal facilities. We also thank Mr. Alan Shipley (Applicable Electronics, LLC.) for lending and assisting with the SVET system, and to Mr. Eric Karplus (Science Wares, Inc.) for software support. Work at E.H.B. lab was funded by an European Research Council (ERC) under the European Union's Horizon 2020 research and innovation programme (grant agreement No. 950254), an EMBO IG Project Number 4765 and a la Caixa Junior Leader Incoming (94978). E.H.B. also acknowledge the support of Instituto Gulbenkian de Ciência and Fundação Calouste Gulbenkian (start-up fund I-411133.01). F.F. was supported by IGC (start-up fund I-411133.01) and an EMBO postdoctoral fellowship (ALTF 27-2020); S.M. was supported by an ERC-StG to E.H.B. and a FCT postdoctoral fellowship (2020.00759.CEECIND).

\section{Author contributions}

F.F. and E.H.B. conceived the project and designed the experiments. F.F. performed most of the experiments and data analyses with help from E.H.B. S.M. performed RNA extractions, RNA-seq, PCR experiments and wrote methods for this part. F.F. and E.H.B wrote the manuscript and prepared all the figures. All the authors edited the manuscript.

Competing financial interests. The authors declare non-financial competing interests.

Supplementary information Supplementary information is included here and videos are available upon request to the corresponding author.

Correspondence and requests for materials should be addressed to E.H.B. 


\section{References}

1. Friedl, P. \& Gilmour, D. Collective cell migration in morphogenesis, regeneration and cancer. Nat. Rev. Mol. Cell Biol. 10, 445-457 (2009).

2. Gilmour, D., Rembold, M. \& Leptin, M. From morphogen to morphogenesis and back. Nature 541, 311-320 (2017).

3. SenGupta, S., Parent, C. A. \& Bear, J. E. The principles of directed cell migration. Nat. Rev. Mol. Cell Biol. 22, 529-547 (2021).

4. Szabó, A. \& Mayor, R. Mechanisms of Neural Crest Migration. Annu. Rev. Genet. 52, 43-63 (2018).

5. Gallik, K. L. et al. Neural crest and cancer: Divergent travelers on similar paths. Mechanisms of Development vol. 148 89-99 (2017).

6. Levin, M. Bioelectric signaling: Reprogrammable circuits underlying embryogenesis, regeneration, and cancer. Cell 184, 1971-1989 (2021).

7. Mayor, R. \& Theveneau, E. The neural crest. Dev. 140, 2247-2251 (2012).

8. Mayor, R. \& Etienne-Manneville, S. The front and rear of collective cell migration. Nature Reviews Molecular Cell Biology vol. 17 97-109 (2016).

9. Bajanca, F. et al. In vivo topology converts competition for cell-matrix adhesion into directional migration. Nat. Commun. 10, 1518 (2019).

10. Mehta, A. S. et al. Physiological electric fields induce directional migration of mammalian cranial neural crest cells. Dev. Biol. 471, 97-105 (2021).

11. Stump, R. F. \& Robinson, K. R. Xenopus Neural Crest Cell Migration in an Applied Electrical Field. J. Cell Biol. 97, 1226-33 (1983).

12. Nuccitelli, R. \& Smart, T. Extracellular Calcium Levels Strongly Influence Neural Crest Cell Galvanotaxis. Biol. Bull. 176, 130-135 (1989).

13. Metcalf, M. E. M., Shi, R. \& Borgens, R. B. Endogenous ionic currents and voltages in amphibian embryos. J. Exp. Zool. 268, 307-322 (1994).

14. Shi, R. \& Borgens, R. B. Three-dimensional gradients of voltage during development of the nervous system as invisible coordinates for the establishment of embryonic pattern. Dev. Dyn. 202, 101-114 (1995).

15. Metcalf, M. E. M. \& Borgens, R. B. Weak applied voltages interfere with amphibian morphogenesis and pattern. J. Exp. Zool. 268, 323-338 (1994).

16. Ferreira, F., Luxardi, G., Reid, B. \& Zhao, M. Early bioelectric activities mediate redoxmodulated regeneration. Development 143, 4582-4594 (2016).

17. McCaig, C. D., Rajnicek, A. M., Song, B. \& Zhao, M. Controlling cell behavior electrically: current views and future potential. Physiol. Rev. 85, 943-978 (2005).

18. Szabó, A., Theveneau, E., Turan, M. \& Mayor, R. Neural crest streaming as an emergent property of tissue interactions during morphogenesis. PLOS Comput. Biol. 15, e1007002 (2019).

19. Theveneau, E. et al. Chase-and-run between adjacent cell populations promotes directional collective migration. Nat. Cell Biol. 2013157 15, 763-772 (2013). 
20. Sroka, J. et al. Lamellipodia and membrane blebs drive efficient electrotactic migration of rat walker carcinosarcoma cells WC 256. PLoS One 11, e0149133 (2016).

21. Rajnicek, A. M., Foubister, L. E. \& McCaig, C. D. Prioritising guidance cues: Directional migration induced by substratum contours and electrical gradients is controlled by a rho/cde 42 switch. Dev. Biol. 312, 448-460 (2007).

22. Zhu, K. et al. Expression of integrins to control migration direction of electrotaxis. FASEB J. 33, 9131-9141 (2019).

23. Huang, Y. J. et al. Cellular microenvironment modulates the galvanotaxis of brain tumor initiating cells. Sci. Rep. 6, 1-10 (2016).

24. Cortese, B., Palamà, I. E., D’Amone, S. \& Gigli, G. Influence of electrotaxis on cell behaviour. Integr. Biol. 6, 817-830 (2014).

25. Nakajima, K. et al. KCNJ15/Kir4.2 couples with polyamines to sense weak extracellular electric fields in galvanotaxis. Nat. Commun. 6, 1-10 (2015).

26. Sun, Y. et al. Infection-generated electric field in gut epithelium drives bidirectional migration of macrophages. PLoS Biol. 17, e3000044 (2019).

27. Zhao, M. et al. Electrical signals control wound healing through phosphatidylinositol-3-OH kinase-gamma and PTEN. Nature 442, 457-60 (2006).

28. Murata, Y., Iwasaki, H., Sasaki, M., Inaba, K. \& Okamura, Y. Phosphoinositide phosphatase activity coupled to an intrinsic voltage sensor. Nature 435, 1239-43 (2005).

29. Ratzan, W. J., Evsikov, A. V, Okamura, Y. \& Jaffe, L. A. Voltage sensitive phosphoinositide phosphatases of Xenopus: their tissue distribution and voltage dependence. J. Cell. Physiol. 226, 2740-2746 (2011).

30. Liu, L. et al. A glutamate switch controls voltage-sensitive phosphatase function. Nat. Struct. Mol. Biol. 19, 633-641 (2012).

31. Hossain, I. et al. Enzyme domain affects the movement of the voltage sensor in ascidian and zebrafish voltage-sensing phosphatases. J. Biol. Chem. 283, 18248-18259 (2008).

32. Theveneau, E. et al. Collective Chemotaxis Requires Contact-Dependent Cell Polarity. Dev. Cell 19, 39-53 (2010).

33. Borgens, R. B., Robinson, K. R., Vanable, J. W. \& McGinnis, M. E. Electric Fields in Vertebrate Repair: Natural and Applied Voltages in Vertebrate Regeneration and Healing. (New York: Alan R. Liss, 1989).

34. Richardson, J., Kotevski, A. \& Poole, K. From stretch to deflection: the importance of context in the activation of mammalian, mechanically activated ion channels. FEBS J. (2021) doi:10.1111/febs.16041.

35. Benko, R. \& Brodland, G. W. Measurement of in vivo stress resultants in neurulation-stage amphibian embryos. Ann. Biomed. Eng. 35, 672-681 (2007).

36. Chen, X. \& Wayne Brodland, G. Multi-scale finite element modeling allows the mechanics of amphibian neurulation to be elucidated. Phys. Biol. 5, 015003 (2008).

37. Jacobson, A. G. \& Gordon, R. Changes in the shape of the developing vertebrate nervous system analyzed experimentally, mathematically and by computer simulation. J. Exp. Zool. 197, 191-246 (1976). 
38. Davey, C. F. \& Moens, C. B. Planar cell polarity in moving cells: Think globally, act locally. Dev. 144, 187-200 (2017).

39. Nishimura, T., Honda, H. \& Takeichi, M. Planar cell polarity links axes of spatial dynamics in neural-tube closure. Cell 149, 1084-1097 (2012).

40. Suchyna, T. M. et al. Identification of a peptide toxin from Grammostola spatulata spider venom that blocks cation-selective stretch-activated channels. J. Gen. Physiol. 115, 583-598 (2000).

41. Koser, D. E. et al. Mechanosensing is critical for axon growth in the developing brain. Nat. Neurosci. 19, 1592-1598 (2016).

42. Zhao, S., Mehta, A. S. \& Zhao, M. Biomedical applications of electrical stimulation. Cell. Mol. Life Sci. 77, 2681-2699 (2020).

43. Barriga, E. H., Franze, K., Charras, G. \& Mayor, R. Tissue stiffening coordinates morphogenesis by triggering collective cell migration in vivo. Nature 554, 523-527 (2018).

44. Chernet, B. T. \& Levin, M. Transmembrane voltage potential of somatic cells controls oncogene-mediated tumorigenesis at long-range. Oncotarget 5, 3287-3306 (2014).

45. Murphy, S. V \& Atala, A. 3D bioprinting of tissues and organs. Nat Biotech 32, 773-785 (2014). 


\section{Methods}

\section{Frog manipulation and embryo generation}

Xenopus laevis (Daudin, 1802) embryos were obtained from in vitro fertilization as previously described ${ }^{46}$. Briefly, injection of human chorionic gonadotropin (MSD Animal Health, Chorulon) into the dorsal lymph sac was used to induce the ovulation in mature females. Upon gentle squeezing, the females laid oocytes that were fertilized using a sperm solution containing $1 / 5$ of freshly collected testis in $500 \mu 1$ of Marc's modified Ringer $0.1 \times$ medium (MMR: $\mathrm{NaCl} 10 \mathrm{mM}, \mathrm{CaCl}_{2} \cdot 2 \mathrm{H}_{2} \mathrm{O} 0.2 \mathrm{mM}, \mathrm{KCl} 0.2 \mathrm{mM}, \mathrm{MgCl}_{2} \cdot 6 \mathrm{H}_{2} \mathrm{O} 0.1 \mathrm{mM}$, and HEPES $0.5 \mathrm{mM}$, adjusted to $\mathrm{pH}$ 7.1-7.2). To collect testes, mature males were dissected after being euthanized in overdose tricaine. Embryos were incubated between 12 and $23{ }^{\circ} \mathrm{C}$ until the desired stages were reached. Xenopus embryos were staged by following established developmental tables ${ }^{47}$. All animal procedures and euthanasia were reviewed and approved by the Ethics Committee and Animal Welfare Body (ORBEA) of the Instituto Gulbenkian de Ciência (IGC) and complied with the Portuguese (Decreto-Lei $n^{\circ} 113 / 2013$ ) and European (Directive 2010/63/EU) legislation.

\section{Microinjection and pharmacologic modulations}

Morpholino, mRNA and/or DNA injections were performed by targeted microinjections using calibrated glass needles that were mounted onto a cell microinjector (MDI, PM1000). Eggs were de-jellied in a cysteine solution ( $1 \mathrm{~g}$ of cysteine and $500 \mu \mathrm{l}$ of $\mathrm{NaOH} 5 \mathrm{M}$ in $50 \mathrm{ml}$ MMR 0.1×) and transferred to ficoll 5\% in MMR 0.45× (w/v) (Sigma-Aldrich, P7798). To target the neural crest population, one dorsal and one ventral animal blastomeres were injected at 8-cell stage (this injection can also target other ectodermal tissues). The neural fold was targeted by injecting one dorsal animal blastomeres at 16-cell stage (Supplementary Fig.. 7). Blastomeres were injected with $10 \mathrm{nl}$ of the specified solution. When required, cells were fluorescently tagged with mRNA of nuclear RFP, membrane RFP and/or membrane GFP; transcripts were generated in vitro by using the mMESSAGE mMACHINE SP6 kit (ThermoFisher, AM1340), according to manufacturer's instructions; and, 250 pg per embryo per construct was injected or co-injected with other treatments. The PCP inhibition was achieved with the injection of $2 \mathrm{ng} \mathrm{DshDEP}^{+}$per embryo. The plasmids for Vsp1-C301S (Addgene, 51882), Vsp1-R152Q-GFP (Addgene, 51884), Vsp1-GFP (Addgene, 51883) and ElectricPkcpEGFP (Addgene, 40314) were previously reported ${ }^{29,48}$. Vsp1-C301S construct was injected 
for a final $1.5 \mathrm{ng}$ per embryo. Vsp1-R152Q-GFP, Vsp1-GFP and ElectricPk-cpEGFP plasmids were transcribed using the mMESSAGE mMACHINE T7 kit (Thermo-Fisher, AM1344) and mRNA was injected for a final $1,1.5$ or 1.5 ng per embryo, respectively.

For drug modulation, the peptide toxin GsMTx4 (Smartox Biotechnology, 08GSM001) was dissolved in $\mathrm{H}_{2} \mathrm{O}$ in a stock of $200 \mu \mathrm{M}$ and stored at $-80{ }^{\circ} \mathrm{C}$. GsMTx4 was reconstituted in MMR $0.1 \times$ and inoculated at a final concentration of $5 \mu \mathrm{M}$. The control of GsMTx 4 was the inoculation of $\mathrm{H}_{2} \mathrm{O}$ diluted in MMR $0.1 \times$.

\section{Neural crest dissection, culture and transplantations}

Cephalic neural crest populations were dissected from embryos at stages 16 and 17 and cultured ex vivo as previously described ${ }^{49}$. Briefly, the vitelline membrane was removed with fine forceps (Dumont, \#4) and the embryos were gently immobilized in modelling clay. A hair knife (eyebrow glued into a glass pipette) was used to remove the epidermis and carefully explant the underlying neural crest. These explants were cut into small clusters and cultured in Danilchik's for Amy 1× medium (DFA: $\mathrm{NaCl} 53 \mathrm{mM}, \mathrm{Na}_{2} \mathrm{CO}_{3} 5 \mathrm{mM}$, potassium gluconate 4.5 $\mathrm{mM}$, sodium gluconate $32 \mathrm{mM}, \mathrm{MgSO}_{4} \cdot 7 \mathrm{H}_{2} \mathrm{O} 1 \mathrm{mM}, \mathrm{CaCl}_{2} \cdot 2 \mathrm{H}_{2} \mathrm{O} 1 \mathrm{mM}$, BSA $0.1 \%(\mathrm{w} / \mathrm{v})$, adjusted to $\mathrm{pH} 8.3$ with Bicine $1 \mathrm{M})$. Clusters were then plated into glass-bottom dishes coated with $62.5 \mu \mathrm{g} \mathrm{ml}^{-1}$ of fibronectin (Sigma-Aldrich, F1141) and let to adhere to the substrate for 15-30 min. Typically, cultures were made from neural crests explanted from 3-6 embryos per condition and arranged in quincunxes.

For in vivo transplantation, appropriate wild-type or injected neural crests from donor and host were explanted as aforementioned. Using the hair knife, the donor neural crest was grafted into the host embryo and held in place by a small piece of coverglass $(\sim 1 \times 1 \mathrm{~mm})$. Once host embryos healed (15-60 min), the glass was removed and embryos were cultured in MMR $0.3 \times$ until the desired stage. For time-lapse imaging, embryos were mounted on agarose dishes with $\sim 1 \mathrm{~mm}$ lanes filled with methyl cellulose $3 \%$ in phosphate-buffered saline (PBS; w/v) to minimize embryo drifting.

\section{Vibrating probe measurements in vivo}

Extracellular net electric current density was measured using a non-invasive vibrating voltage probe as previously described ${ }^{50,51}$. The vector form of Ohm's law, $E F=J_{I} \times \rho$, defines 
current density $\left(J_{I}\right)$ as directly proportional to electric fields $(E F)$, with the resistivity $(\rho)$ of the medium as the constant of proportionality ${ }^{17}$. Hence, electric currents and fields are intrinsically interdependent vector phenomena, with both magnitude and direction.

The measuring probe is a platinum/iridium microelectrode (MicroProbes for Life Science, PI10036.0A10) that was incorporated into the turnkey system Scanning Vibrating Electrode Technique (SVET; Applicable Electronics). Prior to measurements, the probe was platinum-electroplated at the tip ( $\sim 25 \mu$ m diameter) and calibrated with a known electric current density. Briefly, with the probe horizontally vibrating between 100-200 Hz, a glass microelectrode point source positioned $150 \mu \mathrm{m}$ away and passing $60 \mathrm{nA}$ calibrated the vibrating probe in MMR $0.1 \times$ for an electric current density of $21.2 \mu \mathrm{A} \mathrm{cm}^{-2}$.

The SVET system is fitted with a stereoscope that allows to position embryos into a customized non-conductive measuring chamber, containing a plastic lane to hold the embryos in a dorsal up position, allowing perpendicular access of the vibrating probe. The currents were measured 10-30 $\mu \mathrm{m}$ away from the embryo surface and sampled every $1 \mathrm{~s}$ until a plateau was reached (typically in $<1 \mathrm{~min}$ ) (Supplementary Fig.. 1). Reference values were acquired with the probe $>1 \mathrm{~mm}$ away from embryo. As we follow the conventional current, defined by the flow of positive charge, outward current density is the net positive charge exiting the embryo and inward current density is the one entering the embryo. Measurements in the regions and stages specified occurred at $20-22{ }^{\circ} \mathrm{C}$ in MMR $0.1 \times$. Data and metadata were acquired and extracted using the interface software Automated Scanning Electrode Technique (ASET-LV4; Science Wares) and statistically analysed using Excel 2016 (Microsoft) and GraphPad Prism v9.0.0 (GraphPad Software).

\section{Electric field strength estimation}

The imposed EFs magnitude was estimated from the embryo currents that we measured during neurulation. We observed differential current drops (neural fold minus flank non-neural ectoderm) ranging $\sim 0.5-2.5 \mu \mathrm{A} \mathrm{cm}^{-2}$, over an approximate $200-400 \mu \mathrm{m}$ distance (Fig. 1b-d). Following Ohm's law, these currents should generate $\sim-12 \mathrm{mV}$ voltage drops, corresponding to mediolateral endogenous EFs of $\sim 10-60 \mathrm{mV} \mathrm{mm}^{-1}$ detected along the migratory path of neural crest cells. These values fall within previously determined EFs of $\sim 5-75 \mathrm{mV} \mathrm{mm}^{-1}$ in rostral regions of different amphibians ${ }^{13,14}$. Thus, we followed these values in our ex vivo and in vivo electrotaxis assays. 


\section{Electrotaxis assay ex vivo}

The application of exogenous direct current electric fields ${ }^{52}$ was performed in a custommade electrotaxis chamber. The electrotactic chambers with a $22 \times 8 \times 0.3 \mathrm{~mm}$ EFs tunnel were created by placing two single-use strips of polydimethylsiloxane (PDMS) silicone (Sylgard, 184) (0.3 mm height) onto the sides of a reusable 1-well chambered coverglass (Nunc, 155360). For every new assay, the bottom coverglass was replaced by an equivalent coverglass glued with twinsil silicone (Picodent, 1300 1000). To build the PDMS strips, PDMS was prepared by mixing the elastomer/curing agent in a 10:1 ratio. This mix was vacuum degassed and poured into a mould assembled from two silanized microscopic slides spaced by two cover glasses no. 1. The PDMS was cured overnight at $65^{\circ} \mathrm{C}$, manually trimmed and cut into strips. At this stage, neural crest clusters were plated into the fibronectin-coated electrotactic chamber tunnel and let to adhere (Fig. 1e). Then, using electric insulating grease (Dow Corning, DC4), a coverglass roof was attached to the PDMS sides to cover the clusters and electrically seal the EF tunnel. Two dams made of PDMS $(18 \times 2 \times 7 \mathrm{~mm})$ were sealed with grease to the extremes of the roof, generating DFA $1 \times$ medium reservoirs with $3 \mathrm{ml}$ volume capacity. To minimize evaporation, a lid with holes for the agar salt bridges and voltmeter access was used. This setup was mounted for time-lapse microscopy and once all positions were selected, EFs of the above calculated magnitudes were applied. The EFs were channelled through the tunnel via chloritized silver electrodes (Advent, AG549107) and agar salt bridges, composed of agarose 1.5\% in Steinberg's solution (w/v) ( $\mathrm{NaCl} 58 \mathrm{mM}, \mathrm{KCl} 0.67 \mathrm{mM}, \mathrm{MgSO}_{4} \cdot 7 \mathrm{H}_{2} \mathrm{O} 1.3 \mathrm{mM}, \mathrm{CaNO}_{3} \cdot 4 \mathrm{H}_{2} \mathrm{O} 0.44 \mathrm{mM}$, Tris base $4.6 \mathrm{mM}$, adjusted to $\mathrm{pH} 7.4$ ). The required voltages were provided by a direct current power supply (Apelex, 117240 or BK Precision, 9132B) fine-tuned by a resistance decade box (Tenma, 72-7270) and measured in the electrotactic chamber by a voltmeter. Following conventional current, charge flows from anode $(+$ pole $)$ to cathode $(-$ pole $)$.

\section{Application of electric fields in vivo}

To apply parallel or antiparallel exogenous electric fields $\left(100 \mathrm{mV} \mathrm{mm}^{-1}\right)$ along the migratory path of neural crest cells, we used the same power supply, electrodes and agar bridges described in the ex vivo setup, but used a different electric chamber. Briefly, in a Petri dish half filled with modelling clay, a $22 \times 10 \times 1 \mathrm{~mm}$ EFs tunnel was modelled into the centre of the dish. Under a stereoscope, the embryos were gently immobilized in the clay, arranged in quincunxes 
and oriented to maximize the effect of the applied EFs. Coverglass roof, dams and lid were used as in the ex vivo electrotaxis assay. EFs were applied from stage 17 to stage 22 and embryos were immediately fixed and processed for in situ hybridization (as described below).

\section{Motility, protrusion and chemotaxis assays}

To test the basal motility and directionality of wild-type and transfected neural crests, we used motility, protrusion and chemotaxis assays ${ }^{43,49}$. For motility, the neural crest clusters were plated into a plastic-bottom dish (Falcon, 351006) coated with fibronectin $125 \mu \mathrm{g} \mathrm{ml} \mathrm{m}^{-1}$. After adhesion for $1 \mathrm{~h}$, the tight-fit lid was carefully put and the dish was flipped for imaging. For protrusion, the clusters were plated into a fibronectin-coated glass-bottom dish and let to adhere prior to time-lapse microscopy. For chemotaxis, heparin-acrylic beads (Sigma-Aldrich, H5263 (discontinued)) were soaked overnight with human SDF-1 $3.3 \mu \mathrm{g} \mathrm{ml}^{-1}$ (Sigma-Aldrich, S1577) at $4{ }^{\circ} \mathrm{C}$. Using a fibronectin-coated glass-bottom dish (WPI, FD5040), the clusters were placed 2-3 diameters away from the beads that were fixed into a line of grease (Supplementary Fig.. 6).

\section{In situ hybridization}

Embryos were fixed, dehydrated and rehydrated, bleached, hybridized, blocked and stained following in situ hybridization standard protocols ${ }^{49}$. Digoxigenin-labelled antisense probes against complement component $3(c 3)^{53}$ or $s o x 8^{54}$, were used to recognise the neural crest cells in whole embryos. Probes were transcribed with a Riboprobe in vitro Transcription System (Promega, P1420), according to manufacturer's instructions. The signal was revealed using the alkaline phosphatase detection reagents NBT/BCIP (Roche, 11383221001).

\section{In situ hybridisation imaging}

In situ hybridized embryos were mounted on an agarose dish with small depressions to facilitate acquisition. An USB Dino-Eye eyepiece camera (Dino-Lite, AM7025X), mounted onto a stereoscope with cold light, was used to take photomicrographs at $2.5 \times$ magnification, using DinoCapture v2.0 (Dino-Lite). 


\section{sqRT-PCR}

Neural crest explants from pre-migratory stages (17-18) were collected and processed for RNA extraction using the RNeasy Mini kit (Qiagen, 74104). Briefly, 5 ng of isolated total RNA was used as a template for the RT performed using SuperScript IV (Invitrogen, 18090050), according to manufacturer's instructions. To run the PCRs, $2.5 \mu 1$ of resultant cDNA in $25 \mu \mathrm{l}$ of reaction was used for the genes of interest, using Q5 Hot Start High-Fidelity DNA Polymerase (NEB, 174M0493S) with the following primers: vsp1 (FGGCTACGATGACTGTGCCAT; R-TCGACAGTAACAACTTGGTGA); vsp2 (FTCATTGCTTTGTCTCCCTCCC; ACCCTCCTCTTGGTCGTTTT; R-TGACTGAACCCTTCCACGA); ef $1 \alpha$ $(\mathrm{F}-$ CACCAGAACACAGAGTAC; R-TTGGTTTTCGCTGCTTTCT); $k r t 12$ $(\mathrm{F}-$ GAATCATTTGCGCATTTGTGCC; R-CAАССТTCCCATCAACCA); $\quad$ sox8 $(\mathrm{F}-$ GgAgtAAtgAgtgCGACCGA; R-GCCCGACAtGCTCACCTtCA). PCR conditions were as follows: (1) $98{ }^{\circ} \mathrm{C}$ for $30 \mathrm{~s}$ (initial denaturation); (2) $98{ }^{\circ} \mathrm{C}$ for $10 \mathrm{~s}$ (denaturation); (3) $30 \mathrm{~s}$ (annealing) at $64{ }^{\circ} \mathrm{C}(e f 1 \alpha), 60{ }^{\circ} \mathrm{C}(k r t 12), 66{ }^{\circ} \mathrm{C}(\operatorname{sox} 8), 64{ }^{\circ} \mathrm{C}(v s p 1), 68{ }^{\circ} \mathrm{C}(v s p 2)$ or $68{ }^{\circ} \mathrm{C}$ (xbra); (4) $72{ }^{\circ} \mathrm{C}$ for $20 \mathrm{~s}(34 \times$ cycles from step 2 to 4$)$; and, finally, $72{ }^{\circ} \mathrm{C}$ for 2 min for final extension. The amplified fragments were resolved in agarose $0.8 \%(\mathrm{w} / \mathrm{v})$ gel electrophoresis together with $1 \mathrm{~Kb}$ Plus Ladder (Invitrogen, 10787026). While vsp1, vsp2, xbra and sox8 primers were originally designed, efl $\alpha$ and $k r t 12$ primers were previously published ${ }^{55}$.

\section{RNA-seq procedures and analysis}

After assessing the quality of the extracted RNA using HS RNA Screen Tape Analysis (Agilent Technologies), mRNA-library was prepared using an adaptation of the SMARTSeq $2^{56}$. Illumina libraries were performed using an adaptation of the Nextera protocol ${ }^{57}$. The library quantification and quality verification were done using the Fragment Analyzer (AATI) in combination with HS NGS Kit (Agilent Technologies). Sequencing was carried out in NextSeq500 Sequencer (Illumina) using 75 SE high throughput kit. Sequence information was extracted in FastQ format using the bcl2fastq v2.19.1.403 (Illumina).

The RNA-seq analysis was a service conducted by the Bioinformatics Unit at IGC. Briefly, reads from 18S and 28S rRNA were identified and discarded. The good-quality nonribosomal reads (50585 in 51061) were mapped against the reference genome of Xenopus laevis using the annotation XENLA_9.2_Xenbase.gtf (v9.2). The genome FASTA and GTF files were 
downloaded from Xenbase. The gene expression tables were imported into the R v3.6.3 (The $\mathrm{R}$ Foundation for Statistical Computing) to normalize gene expression with the TMM (Trimmed Mean of M-values) procedure ${ }^{58,59}$ with the NOISeq R package (v2.30.0) ${ }^{60}$.

\section{Localization and activation of Vsp1}

To assess the cellular localization and activity of Vsp1, we injected Vsp1-GFP and ElectricPk-cpEGFP, respectively. For localization, neural crest clusters were injected with Vsp1-GFP as described above and plated into fibronectin-coated glass-bottom dishes (WPI, FD35) and let to adhere prior to confocal microscopy. To report Vsp1 response to electric fields we used the Vsp1-based sensor ElectricPk-cpEGF. This sensor was co-injected with the membrane RFP as described above and the expressing clusters without application of EFs or exposed to EFs of $100 \mathrm{mV} \mathrm{mm}^{-1}$ for $1 \mathrm{~h}$ were fixed overnight in formaldehyde $3.7 \%$ in PBS (v/v). After fixation, clusters were washed with PBS and mounted with mowiol (Millipore, 475904) and sealed with nail polish for confocal microscopy.

\section{Cryosectioning}

Embryo sectioning was performed as previously described ${ }^{43}$. Briefly, neural foldinjected embryos were fixed for $2 \mathrm{~h}$ at room temperature and washed twice with phosphate buffer $1 \times\left(\mathrm{NaH}_{2} \mathrm{PO}_{4} \cdot \mathrm{H}_{2} \mathrm{O} 0.2 \mathrm{M}\right.$ and $\mathrm{K}_{2} \mathrm{HPO}_{4} 0.2 \mathrm{M}$, pH 7.4) for 5 min each wash. Embryos were passed to sucrose $15 \%$ in phosphate buffer $(\mathrm{w} / \mathrm{v})$ for $2 \mathrm{~h}$ at room temperature. Embryos were then embedded and orientated in a gelatine solution (gelatine $8 \%$ in $15 \%$ sucrose in phosphate buffer, w/v) and incubated for $1 \mathrm{~h}$ at $42{ }^{\circ} \mathrm{C}$. Gelatine blocks were flash frozen at -80 ${ }^{\circ} \mathrm{C}$ in pre-cooled heptane and sectioned in $25 \mu \mathrm{m}$ slices in a cryostat (Leica, CM3050 S). The slides dried overnight at room temperature and were mounted with mowiol and sealed with nail polish prior to confocal microscopy.

\section{Laser ablation assay}

Recoil velocity as a proxy of membrane tension build-up or stretching was assessed using laser ablation experiments ${ }^{61}$. The centre of fluorescently labelled and mediolaterally oriented membrane junctions (i.e., perpendicular to the neural fold) was photoablated using a MicroPoint system (Andor) fitted with a $350 \mathrm{~nm}$ pulsed laser and 365/435 dye. The system was 
controlled by Metamorph software (Molecular Devices). Junctions were ablated using one pulse of $5-10 \%$ laser at $21-22{ }^{\circ} \mathrm{C}$ after the first intact image was acquired. Up to 3 ablations were performed per embryo, with sufficient distance from each other to avoid the effect of local membrane relaxation.

\section{Time-lapse imaging and confocal laser scanning}

Graft experiments. Grafted embryos were $z$-stacked (10 $\mu \mathrm{m}$ sections) and imaged using 488 and $561 \mathrm{~nm}$ laser lines on an Imager Z2/ApoTome.2 system (Zeiss) equipped with an Orca Flash 4.0 v2 CMOS camera (Hamamatsu), or on a SP5 confocal system (Leica) with spectral detection. Water immersion $10 \times$ objectives were used in both systems: N-Achroplan $10 \times / 0.30$ NA (Zeiss) and HC APO L 10×/0.30 NA (Leica), respectively. Microscopes (both upright setups) were controlled by ZEN v3.1 (Zeiss) or LAS AF (Leica), respectively. Recording started at around stage 20 and was acquired every 5 or $10 \mathrm{~min}$ for up to $7 \mathrm{~h}$ at $21-22^{\circ} \mathrm{C}$.

Electrotaxis. Cell migration of neural crest clusters was recorded on a Nikon HCS microscope, equipped with a Zyla 4.2 sCMOS camera (Andor) and using a PL APO 10×/0.45 NA objective (Nikon). The system was controlled by NIS-Elements (Nikon) to acquire timelapse microscopy every $5 \mathrm{~min}$ for up to $10 \mathrm{~h}$ at $18{ }^{\circ} \mathrm{C}$, using 488 and $561 \mathrm{~nm}$ filter sets.

Motility. The inverted dish was mounted in the ApoTome system and clusters were imaged using the EC Plan-Neofluar $10 \times / 0.30$ NA objective by time-lapse mode every 5 min for up to $8 \mathrm{~h}$ at $21-22^{\circ} \mathrm{C}$.

Protrusions. The clusters were imaged using the SP5 confocal system with the HC PL APO 40×/0.80 NA water immersion objective (Leica). Recording occurred in time-lapse mode every 30 or $60 \mathrm{~s}$ for up to $30 \mathrm{~min}$ at $21-22{ }^{\circ} \mathrm{C}$.

Chemotaxis. Cell migration was imaged using the SP5 confocal system or a Thunder Imager 3D Cell Culture system (Leica) with the HC PL APO 10×/0.45 NA objective (Leica), in time-lapse mode every $5 \mathrm{~min}$ for up to $6 \mathrm{~h}$ at $18-22^{\circ} \mathrm{C}$.

Laser ablation. Embryos were imaged using a HC PL APO 63×/1.30 NA glycerine immersion objective (Leica) mounted in an inverted confocal microscope (Yokogawa CSU-X Spinning Disk confocal, onto a Leica DMi8 microscope body). The system was controlled by Metamorph to acquire time-lapse imaging every 2 or $5 \mathrm{~s}$ for up to $30 \mathrm{~s}$, using 488 and $561 \mathrm{~nm}$ laser lines and an iXon Ultra EMCCD camera (Andor). 
Vsp1-GFP imaging. Injected clusters were imaged using an inverted confocal system (Zeiss, LSM 980), equipped with two PMT and one GaAsP and controlled by ZEN Blue v3.0 (Zeiss). Using the 488 and $561 \mathrm{~nm}$ laser lines, a $z$-stack (1 $\mu \mathrm{m}$ sections) was acquired at 21-22 ${ }^{\circ} \mathrm{C}$ with the C Plan-Apochromat $63 \times / 1.40$ NA oil immersion objective (Zeiss).

ElectricPk-cpEGFP imaging. Injected clusters were imaged using an upright confocal system (Leica, Stellaris 5), equipped with Power HyD S detectors and controlled by LAS X (Leica). Using the 488 and $561 \mathrm{~nm}$ laser lines, a $z$-stack ( $1 \mu \mathrm{m}$ sections) was acquired at 21-22 ${ }^{\circ} \mathrm{C}$ with the HC PL APO $63 \times / 1.40$ NA oil immersion objective (Leica).

Neural fold-targeted injection imaging. Embryos of targeted injections of $\mathrm{DshDEP}^{+}$into the neural fold were imaged using the Stellaris 5 confocal system with the HC APO L $10 \times / 0.30$ NA water immersion objective (Leica). The sections of these embryos were imaged using the LSM 980 confocal system with the LD LCI Plan-Apochromat 25×/0.80 NA oil immersion objective (Zeiss).

\section{Cell motility analysis}

Cluster and cell migration. The centre of ex vivo clusters and in vivo cells were tracked typically for 3-6 h (exact time in figure legends) using the Manual Tracking plug-in in Fiji (https://imagej.net/Fiji; v1.53k). The cluster/cell directedness and velocity were computed using the Chemotaxis and Migration Tool v2.0 (Ibidi). Directedness is represented by the forward migration index (FMI), which shows how efficiently cells migrate forwardly parallel to the EFs vector ex vivo or mediolateral axis in vivo. The frequency of angles of the clusters/cells in relation to the EFs vector was computed using Rozeta v2.0 (freeware developed by Jacek Pazera). The anode was set at $180^{\circ}$. The frequency (percentage) of anodal migration was determined by counting the clusters that experienced a biased displacement of the whole cluster diameter towards the anode. In control conditions under EFs, clusters typically displaced several diameters (Supplementary Fig.. 2, Supplementary Video 1,2).

Collective vs. single cell migration. Electrotaxis time-lapses, from independent experiments, where several single cells detached from the migrating collective (cluster) were selected for comparative tracking. The centre of the cluster or single cells were tracked for $1 \mathrm{~h}$. Tracking and analysis were performed as aforementioned. 
Single cell motility. The cluster was let to disperse to allow the appearance of many motile single cells. Single cells were tracked for $1 \mathrm{~h}$ (Supplementary Fig.. 5) and analysed as above.

Cell protrusions. Maximum protrusion area was measured in Fiji using the freehand selection tool (Supplementary Fig.. 5). Protrusion dynamics correspond to the time for a complete cycle of protrusion expansion and retraction. Maximum protrusion area was determined at the maximum protrusion expansion frame.

\section{Neural crest migration analysis}

The frequency of in vivo dCCM was determined by counting the in situ hybridized embryos which formed stereotypical streams (a hallmark of $\mathrm{dCCM}^{18,19}$ ). In control conditions, majority of embryos formed three well-defined streams (Fig. 1a,k). The displacement of neural crest cells was derived with the ratio between the stream length and dorsoventral length of the embryo. All ratios were normalized against the maximum length of the control stream. Lengths were obtained in Fiji using the measurement tool.

\section{Membrane ablation analysis}

The right and left vertexes of fluorescently labelled membranes were tracked for up to 20 s post photoablation using the Manual Tracking plug-in in Fiji (Supplementary Fig.. 8). The recoil velocities were averaged using Excel and plotted with GraphPad Prism.

\section{Image and video treatment}

Substacking, z-projections (maximum intensity), rotations, time colour-coded projections, and time-lapse videos were performed in Fiji. General treatment, including adjustment of contrast and brightness, resizing, pseudocolouring, addition of scales and overlay of text in images and videos were conducted in Fiji and/or Photoshop 2021 (Adobe). Some of the time-lapses, particularly from grafts, were stabilized using the StackReg registration plugin in Fiji. In time colour-coded projections, the background with debris and dead cells was coloured in black in Fiji, for clarity purposes and without interfering with the sample itself. 


\section{Statistical analysis}

The experimenters were not blinded in data acquisition, treatment, or statistical analysis due to the nature of the experimental procedures and the clear embryonic and cellular phenotypes of some manipulations. The embryos were randomly allocated to the specified experimental conditions. Data exclusion criteria were in place to filter out unviable, overaged, misinjected and contaminated embryos and/or cell clusters. Data outliers were removed based on the biological context when flagged using the statistical Grubbs' test and/or ROUT method computed using GraphPad Prism. Majority of datasets presented no statistical outliers. The appropriate inferential statistics were computed using GraphPad Prism and are annotated in the figure legends. For parametric tests, pre-tests were conducted for the assumptions of normality (Kolmogorov-Smirnov and/or Shapiro-Wilk tests) and equal variances (F test). Majority of datasets verified these assumptions. To address non-verified assumptions, we used the Welch's correction or a non-parametric equivalent test. Data representation and sample size (n, biological replicates) are annotated in figures legends. At least three independent experiments were performed per physiological readout, using embryo batches from different females. Differences were significant when the two-tailed $p$ value was $\leq 0.05$ with the following levels of significance: $p>0.05$, non-significant, ${ }^{*} p \leq 0.05, * * p<0.01, * * * p<0.001$, and $* * * * p<$ 0.0001. Data analysis and visualization were performed using Excel, ASET, Chemotaxis and Migration Tool, Rozeta, R, GraphPad Prism, Fiji, Photoshop and Illustrator 2021 (Adobe).

\section{Data and code availability}

The data supporting the findings of this study are embedded in the main text and supplementary information. In addition, other relevant data are available and unrestricted from the corresponding author upon reasonable request. 


\section{References}

46. Sive, H. L., Grainger, R. M. \& Harland, R. M. Xenopus laevis In Vitro Fertilization and Natural Mating Methods. Cold Spring Harb. Protoc. (2007) doi:10.1101/pdb.prot4737.

47. Nieuwkoop, P. D. \& Faber, J. Normal table of Xenopus laevis (Daudin). (Amsterdam: NorthHolland, 1967).

48. Barnett, L., Platisa, J., Popovic, M., Pieribone, V. A. \& Hughes, T. A Fluorescent, GeneticallyEncoded Voltage Probe Capable of Resolving Action Potentials. PLoS One 7, e43454 (2012).

49. Barriga, E. H., Shellard, A. \& Mayor, R. In Vivo and In Vitro Quantitative Analysis of Neural Crest Cell Migration. in Neural Crest Cells: Methods and Protocols, Methods in Molecular Biology (eds. Schwarz, Q. \& Wiszniak, S.) vol. 1976 135-152 (Humana Press, New York, NY, 2019).

50. Reid, B., Nuccitelli, R. \& Zhao, M. Non-invasive measurement of bioelectric currents with a vibrating probe. Nat. Protoc. 2, 661-669 (2007).

51. Shipley, A. M. \& Feijó, J. A. The use of the vibrating probe technique to study steady extracellular currents during pollen germination and tube growth. in Fertilisation in Higher Plants: molecular and cytological aspects 235-250 (1999).

52. Song, B. et al. Application of direct current electric fields to cells and tissues in vitro and modulation of wound electric field in vivo. Nat. Protoc. 2, 1479-1489 (2007).

53. McLin, V. A., Hu, C. H., Shah, R. \& Jamrich, M. Expression of complement components coincides with early patterning and organogenesis in Xenopus laevis. Int. J. Dev. Biol. 52, 1123-1133 (2008).

54. O’Donnell, M., Hong, C. S., Huang, X., Delnicki, R. J. \& Saint-Jeannet, J. P. Functional analysis of Sox8 during neural crest development in Xenopus. Development 133, 3817-3826 (2006).

55. Hong, C. S. \& Saint-Jeannet, J. P. The activity of Pax3 and Zic1 regulates three distinct cell fates at the neural plate border. Mol. Biol. Cell 18, 2192-2202 (2007).

56. Macaulay, I. C. et al. Separation and parallel sequencing of the genomes and transcriptomes of single cells using G\&T-seq. Nat. Protoc. 11, 2081-2103 (2016).

57. Baym, M. et al. Inexpensive Multiplexed Library Preparation for Megabase-Sized Genomes. PLoS One 10, e0128036 (2015).

58. Robinson, M. D. \& Oshlack, A. A scaling normalization method for differential expression analysis of RNA-seq data. Genome Biol. 11, R25 (2010).

59. Robinson, M. D., McCarthy, D. J. \& Smyth, G. K. edgeR: a Bioconductor package for differential expression analysis of digital gene expression data. Bioinformatics 26, 139-140 (2010).

60. Tarazona, S. et al. Data quality aware analysis of differential expression in RNA-seq with NOISeq R/Bioc package. Nucleic Acids Res. 43, e140 (2015).

61. Scarpa, E., Finet, C., Blanchard, G. B. \& Sanson, B. Actomyosin-Driven Tension at Compartmental Boundaries Orients Cell Division Independently of Cell Geometry In Vivo. Dev. Cell 47, 727-740.e6 (2018). 
a<smiles></smiles>

neural crest $\square$ head mesoderm ectoderm vibrating neural plate probe

b

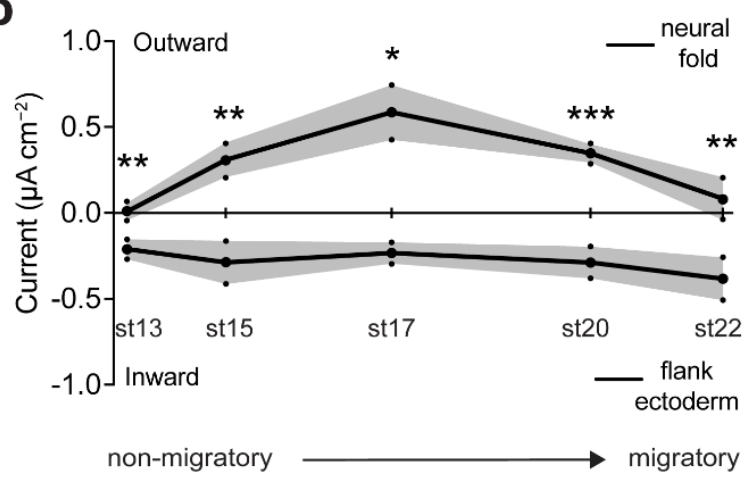

e

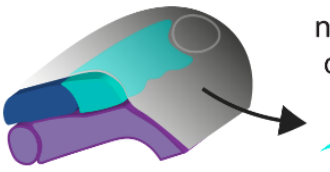

neural

f
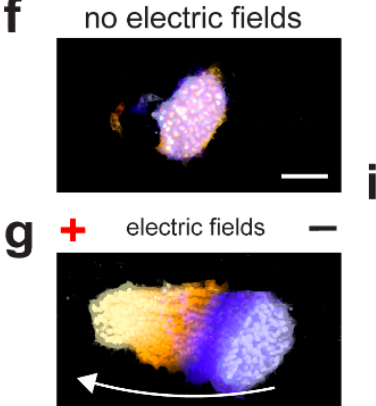

h

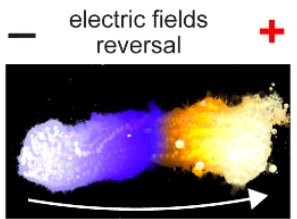

+ electric field $-\mathbf{j}$

0 time in hours 4 non-migratory (stage 13)
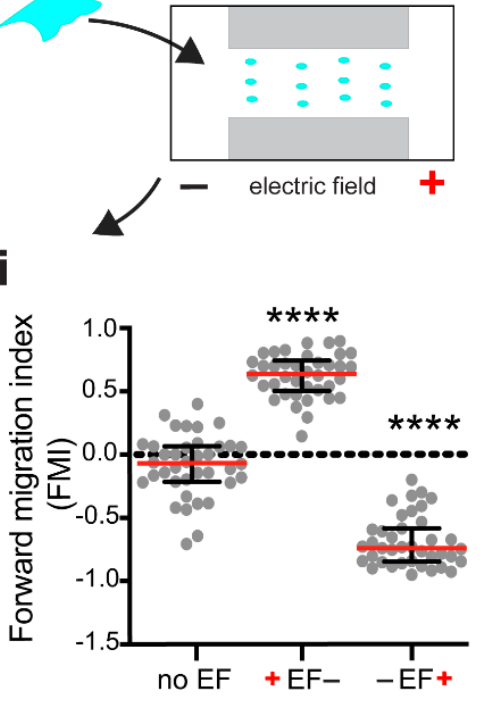

pre-migratory (stage 17.5)

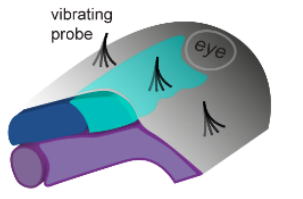

migratory (stage 22)

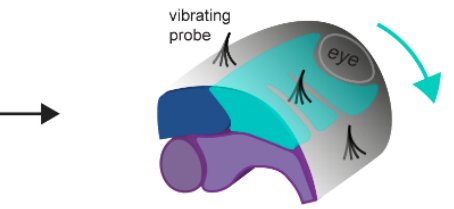

12 hours@18 ${ }^{\circ} \mathrm{C}$
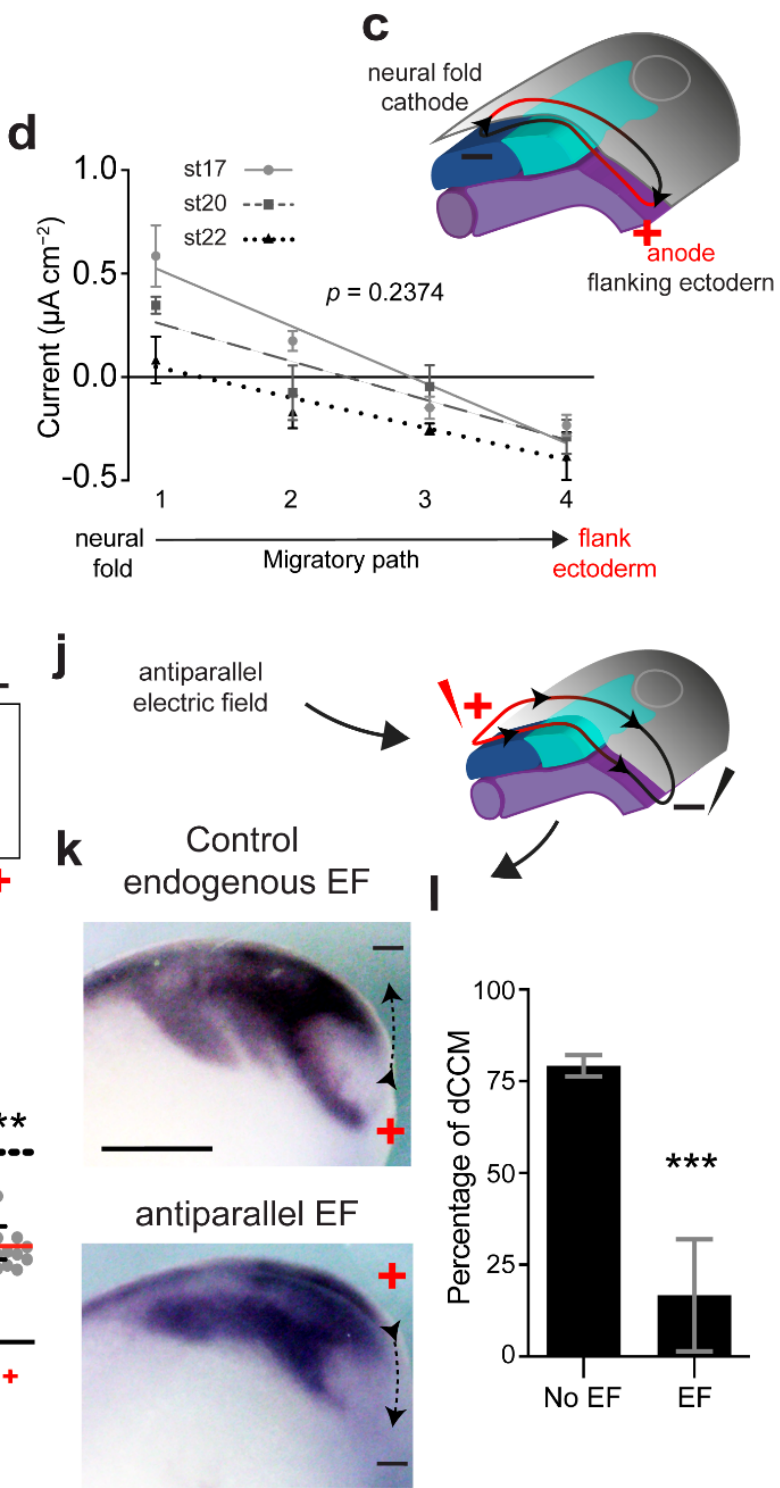

Fig. 1 | Endogenous electric fields modulate neural crest directed collective cell migration.

a, Scheme shows neural crest development and vibrating probe measurements along its migratory path (AP, anteroposterior; ML, mediolateral; DV, dorsoventral). b-d, Mediolateral endogenous electric fields (EFs) emerge in the migratory path of neural crest cells. b, Vibrating probe measurements results; dots represent mean and shade the standard error, paired $t$-test (st13-20) or Wilcoxon test (st22) (both two-tailed), ${ }^{* *} p_{\text {st13 }}=0.0017,{ }^{*} p_{\text {st15 }}=0.0042,{ }^{*} p_{\text {st17 }}=$ 
$0.0339, * * * p_{\mathrm{st} 20}=0.0008, * * p_{\mathrm{st} 22}=0.0078, n=35$ embryos. c, Sustained EF dipole along the migratory path of neural crest cells, the cathode $(-)$ is in the neural folds and the anode $(+)$ in the flanking non-neural ectoderm. d, Linear regressions showing similar slopes of current along the neural crest migratory path at different stages, $p=0.2374$ (two-tailed), $n=23$ embryos. $\mathbf{e}-$ i, Neural crest explants exposed to EFs ex vivo. e, Ex vivo electrotaxis assay diagram. $\mathbf{f}-\mathbf{h}$, Time colour-coded trajectories of neural crest cells migrating in the absence of EFs for $1 \mathrm{~h}(\mathbf{f})$, under EFs $\left(100 \mathrm{mV} \mathrm{mm}^{-1}\right)$ for $4 \mathrm{~h}(\mathbf{g})$ or after EFs reversal for $4 \mathrm{~h}(\mathbf{h})$. White arrows indicate direction of neural crest migration (see Supplementary Video 1). Scale bar, $100 \mu \mathrm{m}$. i, Forward migration index (FMI) quantifications, conditions as indicated. Red lines represent median and error bars the interquartile ranges, paired $t$-test, $* * * * p<0.0001$ (two-tailed), $n=39$ clusters. $\mathbf{j}-$ 1, Antiparallel EFs in vivo alter the pattern of neural crest dCCM. j, Antiparallel EFs application $\left(100 \mathrm{mV} \mathrm{mm}^{-1}\right)$. $\mathbf{k}$, In situ hybridisations showing lateral views of embryos hybridised with $c 3$ (a marker of neural crest migration). Scale bar, $200 \mu \mathrm{m}$. 1, Percentage of embryos displaying streams, column bars represent mean and error bars the standard deviation, Fisher's exact test, $* * * p=0.0002$ (two-tailed), $n_{\mathrm{No}} \mathrm{EFs}=30$ embryos, $n_{\text {Antiparallel } \mathrm{EFs}}=18$ embryos. $\mathbf{f}-\mathbf{h}, \mathbf{k}$, Representative examples from at least three independent experiments; $\mathrm{CI}=95 \%$. 
a
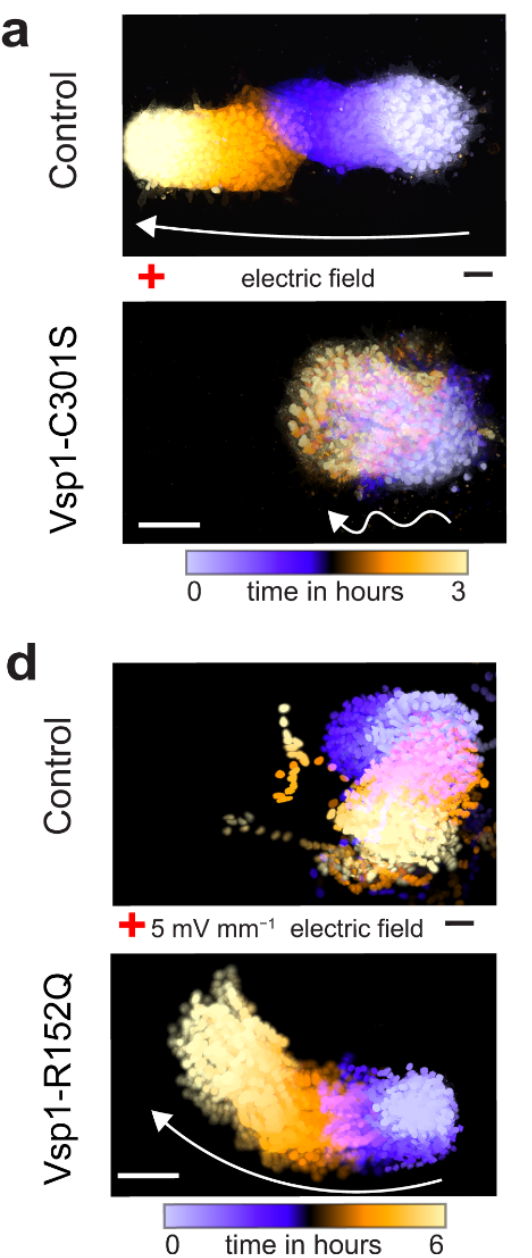

g

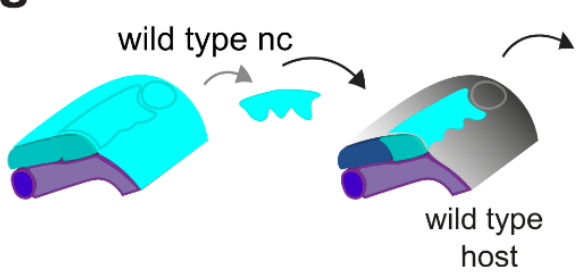

in vivo

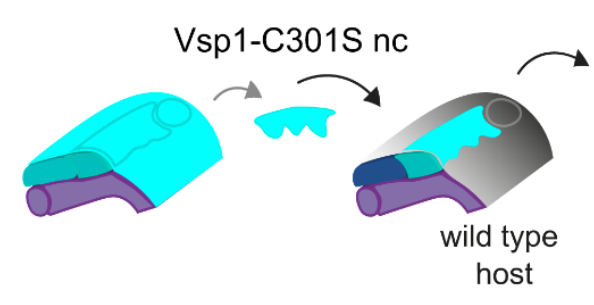

b

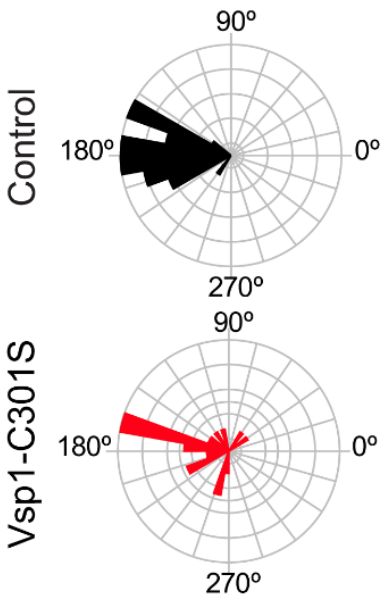

e

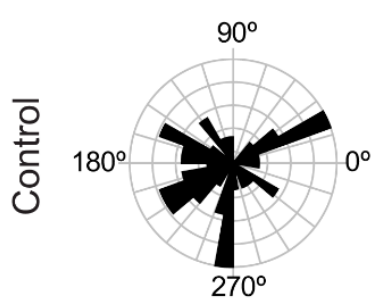

$90^{\circ}$

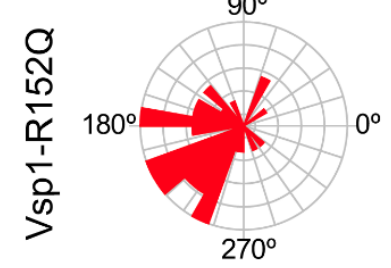

h
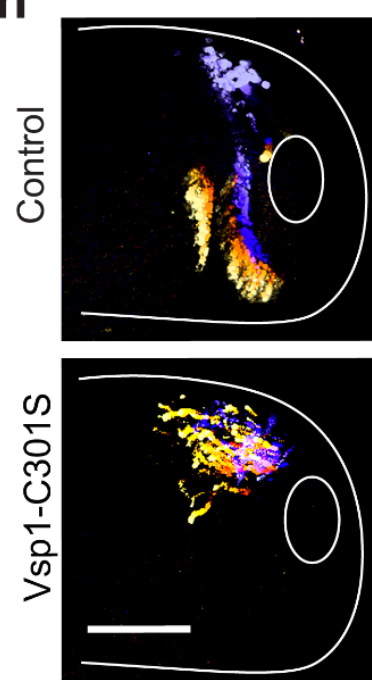

C

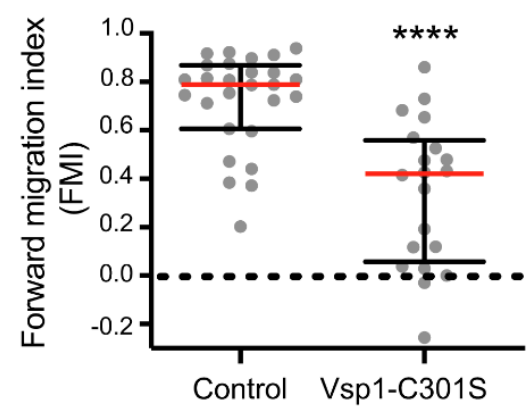

f

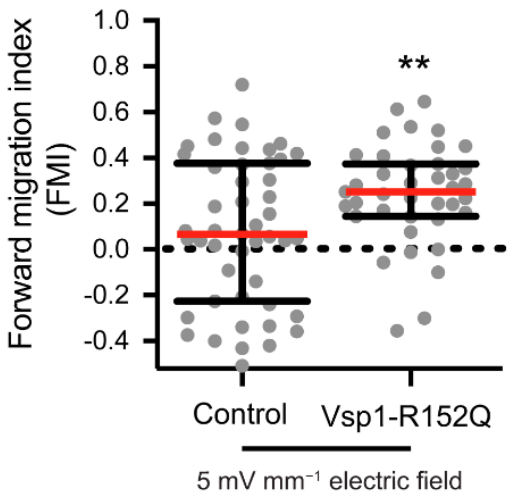

i
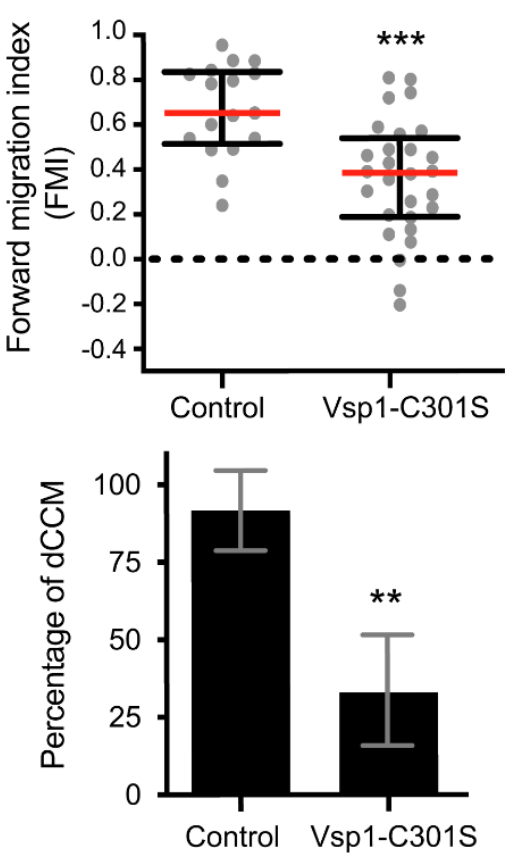

Fig. 2 | Voltage-sensitive phosphatase 1 (Vsp1) controls neural crest directionality. a-c, Effect of Vsp1 (Vsp1-C301S) in neural crest dCCM ex vivo. a, Time colour-coded trajectories of neural crest cells ex vivo, white arrows indicate direction of neural crest migration (see Supplementary Video 5). Scale bar, $100 \mu \mathrm{m}$. b, Rose plots showing the angle frequencies of neural crest migration in relation to the electric field (EF) vector (anode at $180^{\circ}$ ). $\mathbf{c}$, Forward 
migration index (FMI) quantifications, red lines represent median and error bars the interquartile ranges, Mann Whitney $U$-test, $* * * * p<0.0001$ (two-tailed), $n_{\text {Control }}=27$ clusters, $n_{\text {Vsp1-C301S }}=20$ clusters. d-f, Effect of Vsp1 (Vsp1-R152Q) in neural crest directionality exposed to suboptimal EFs ex vivo. d, Time colour-coded trajectories of neural crest cells (see Supplementary Video 6). Scale bar, $100 \mu \mathrm{m}$. e, Rose plots showing the angle frequencies of neural crest migration in relation to the vector of suboptimal EFs (anode at $180^{\circ}$ ). f, FMI quantifications, red lines represent median and error bars the interquartile ranges, $t$-test with Welch's correction, ${ }^{* *} p=0.0069$ (two-tailed), $n_{\text {Control }}=47$ clusters, $n_{\mathrm{Vsp} 1-\mathrm{R} 152 \mathrm{Q}}=39$ clusters. $\mathbf{g}-\mathbf{j}$, Effect of Vsp1-C301S in neural crest dCCM in vivo. $\mathbf{g}$, Scheme of the transplantation assays (nc, neural crest). h, Time colour-coded trajectories of neural crest cells in vivo (lateral view of embryo) (see Supplementary Video 7). Scale bar, $200 \mu \mathrm{m}$. i, FMI quantifications, red lines represent median and error bars the interquartile ranges, $t$-test with Welch's correction, $* * * p=0.0002$ (two-tailed), $n_{\text {Control }}=17$ embryos, $n_{\mathrm{V} \text { sp1-C301S }}=28$ embryos. $\mathbf{j}$, Percentage of embryos displaying streams, column bars represent mean and error bars the standard deviation, Fisher's exact test, ${ }^{*} p=0.0016$ (two-tailed), $n_{\text {Control }}=17$ embryos, $n_{\mathrm{Vsp1-C} 301 \mathrm{~S}}=29$ embryos. $\mathbf{a}, \mathbf{d}, \mathbf{h}$, Representative examples from at least three independent experiments; $\mathrm{CI}=95 \%$. 
a

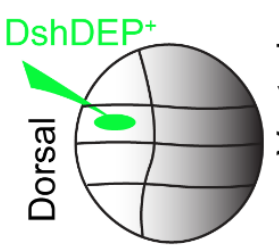

16-cell stage animal view

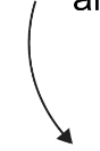

C

e<smiles>[M]C(C)(C)[18O]</smiles>

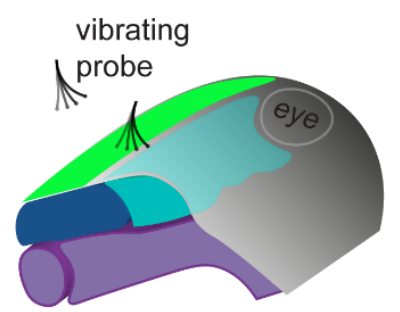

GsMT×4 incubation

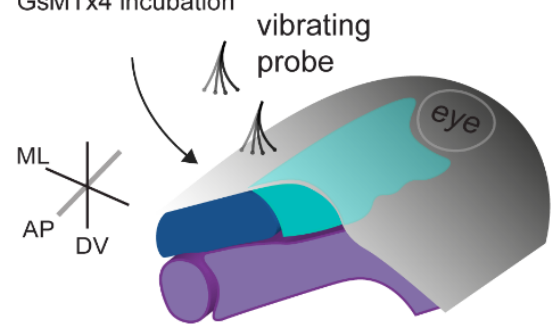

g
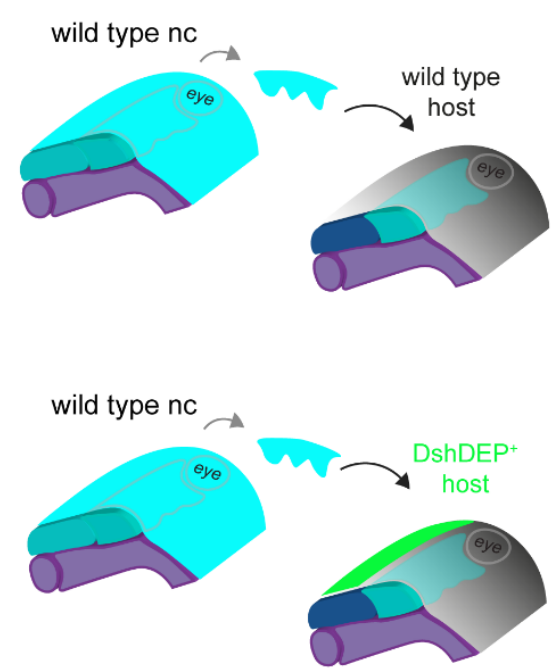

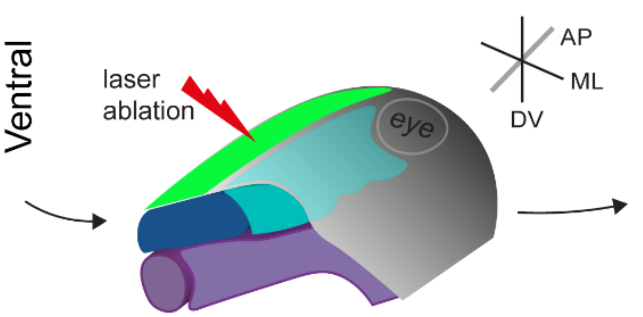

$\square$ neural crest $\square$ neural plate neural fold $\square$ head mesoderm $\square$ ectoderm b

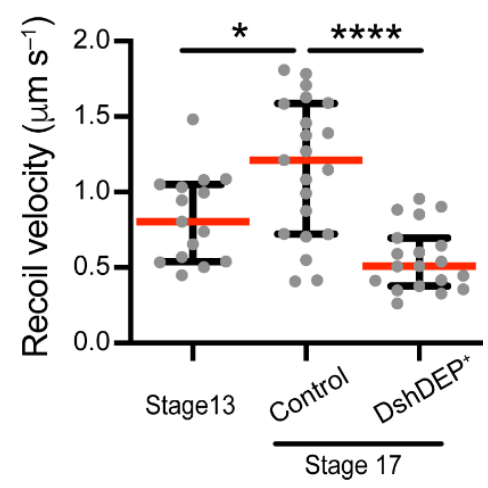

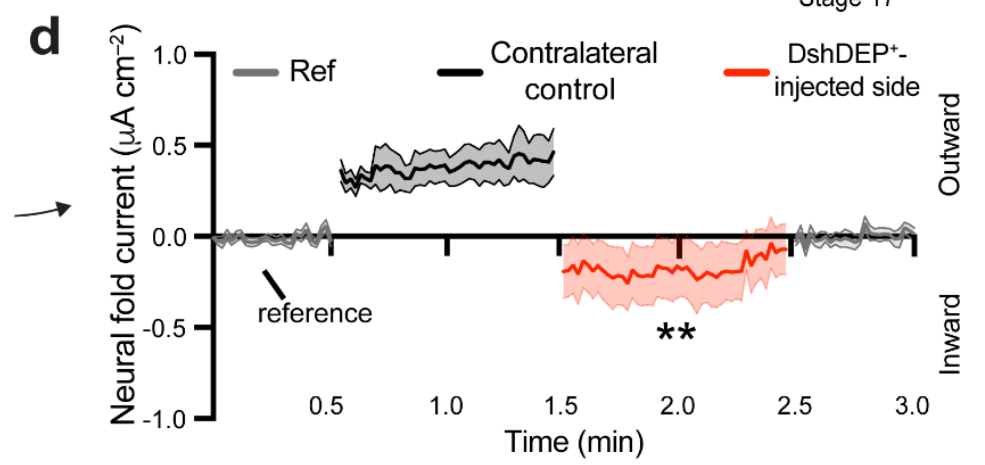

f

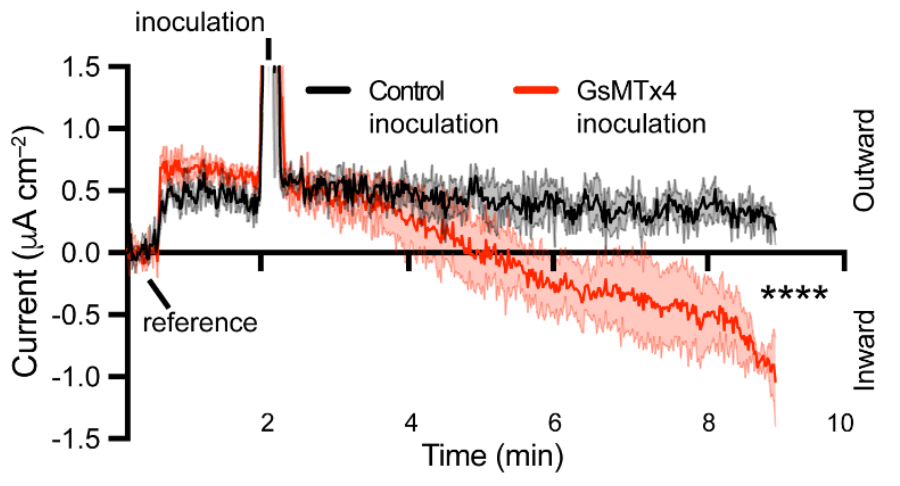

h
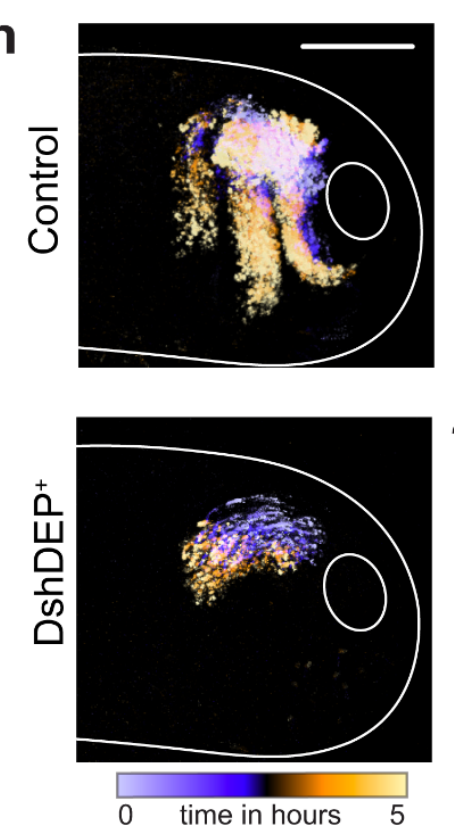

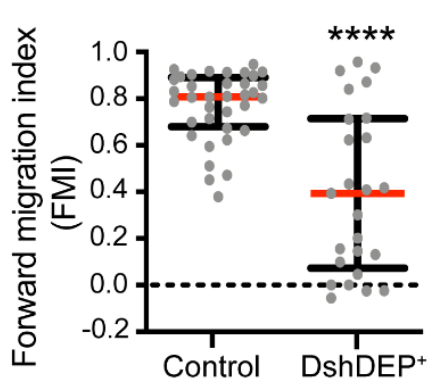

j

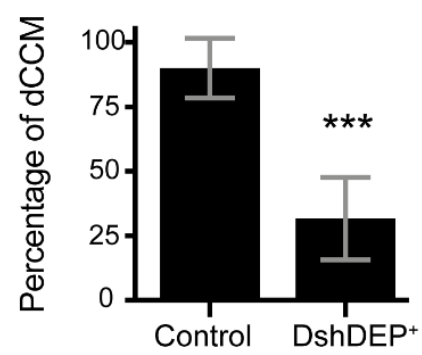


Fig. 3 | Electric fields emerge from PCP-dependent neural fold membrane stretching to guide dCCM in vivo. a, Scheme of $\mathrm{DshDEP}^{+}$neural fold-targeted injection for subsequent laser ablation. (b) Neural fold membrane tension at non-migratory (st13) and pre-migratory (st17, control or $\mathrm{DshDEP}^{+}$neural fold injected) stages. Red lines represent median and error bars the interquartile ranges, Student's $t$-test, ${ }^{*} p=0.0180$ (two-tailed), $t$-test with Welch's correction, $* * * * p<0.0001$ (two-tailed); $n_{\text {Non-migratory }}=15$ cell membranes, $n_{\text {Control }}=21$ cell membranes, $n_{\text {DshDEP }^{+}}=19$ cell membranes. $\mathbf{c}$, Scheme of vibrating probe measurement in $\mathrm{DshDEP}^{+}$neural fold injected embryos. d, Impact of $\mathrm{DshDEP}^{+}$neural fold targeted injections in electric currents, solid lines represent mean and shades the standard error, paired $t$-test (matched means of individual scatter lines), ${ }^{* *} p=0.0019$ (two-tailed), $n=14$ embryos. e, Scheme showing the simultaneous GsMTx4 inoculation and electric currents measurements. $\mathbf{f}$, Impact of Control or GsMTx4 $(5 \mu \mathrm{M})$ inoculation during live recording of currents in the neural fold. (reference, probe away from embryo). Solid line represents mean and shades the standard error, Wilcoxon matched-pairs signed rank test (time-matched after inoculation), ${ }^{* * * *} p<$ $0.0001, n=6$ embryos. $\mathbf{g}-\mathbf{j}$, PCP inhibition in the neural fold impairs neural crest directionality. $\mathbf{g}$, Scheme of the transplantation assays (nc, neural crest). $\mathbf{h}$, Time colour-coded trajectories of neural crest cells (lateral view of embryos) (see Supplementary Video 10). Scale bar, $200 \mu \mathrm{m}$. i, Forward migration index (FMI) quantifications, red lines represent median and error bars the interquartile ranges, Mann Whitney $U$-test, $* * * * p<0.0001$ (two-tailed), $n_{\text {Control }}=36$ cells, $n_{\text {DshDEP }^{+}}=25$ cells. $\mathbf{j}$, Percentage of embryos displaying streams, column bars represent mean and error bars the standard deviation, Fisher's exact test, ${ }^{* * *} p=0.0002$ (two-tailed), $n_{\text {Control }}=$ 19 embryos, $n_{\text {DshDEP }^{+}}=18$ embryos. $g$, Representative examples from at least three independent experiments; $\mathrm{CI}=95 \%$. 
a

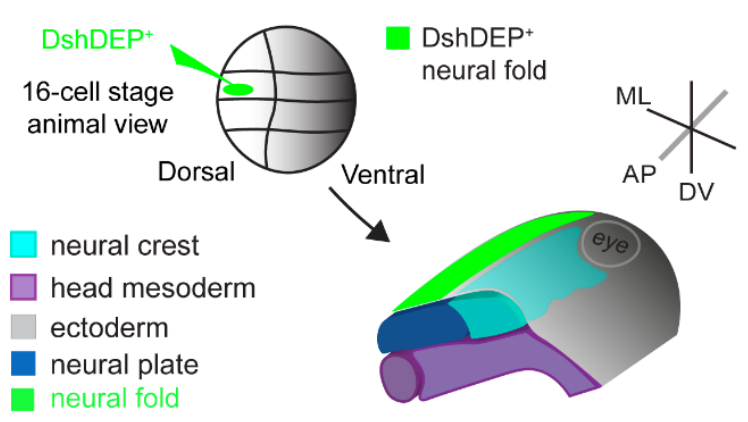

b

no parallel electric field

Control

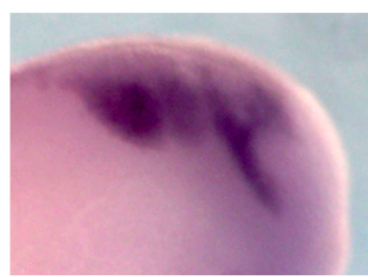

e

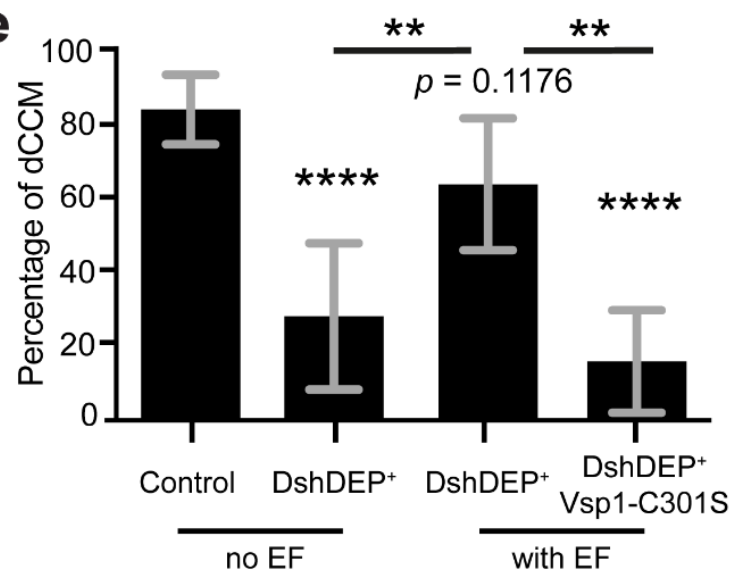

g

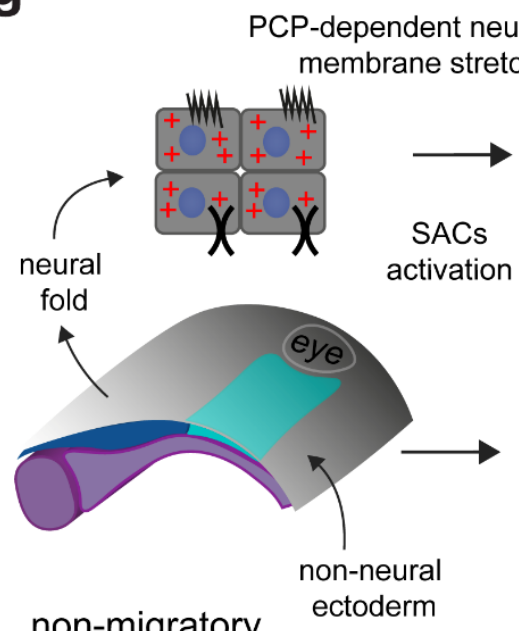

(stage 13)
DshDEP+ $^{+}$

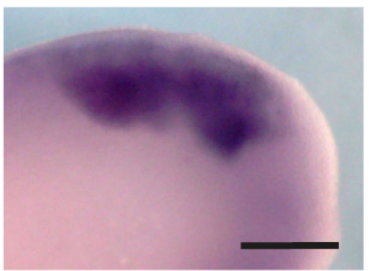

f
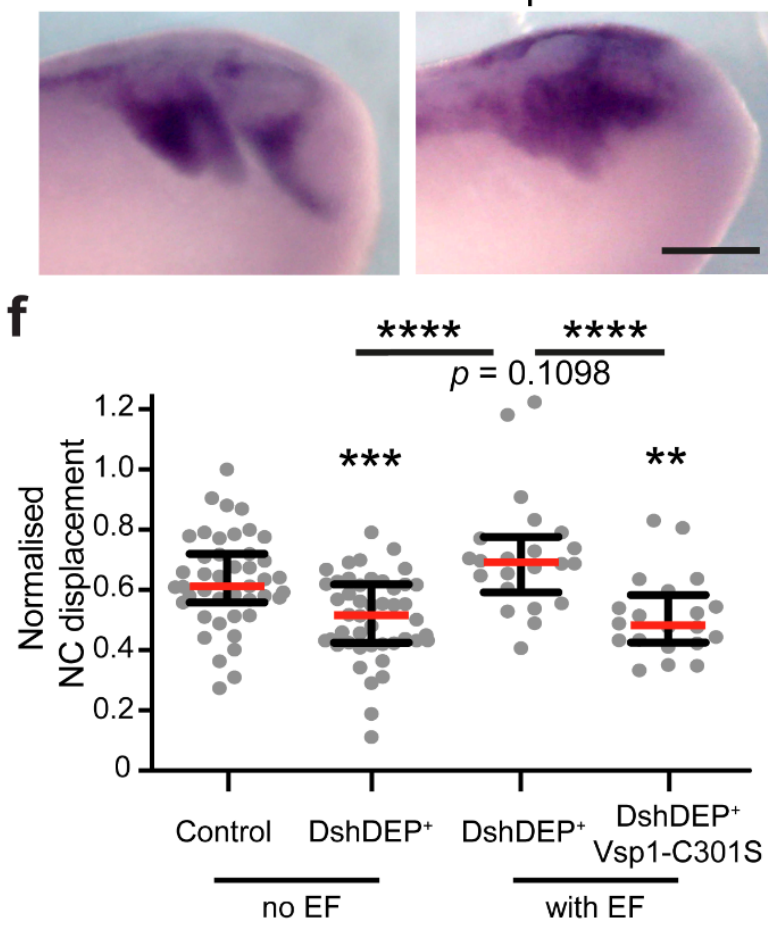

C

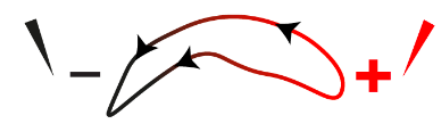

parallel paralle

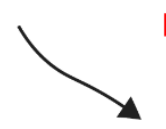

Vsp1-C301S neural crest

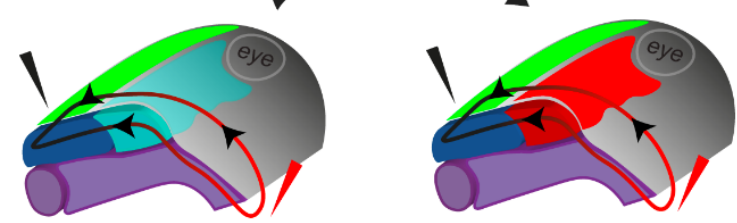

d
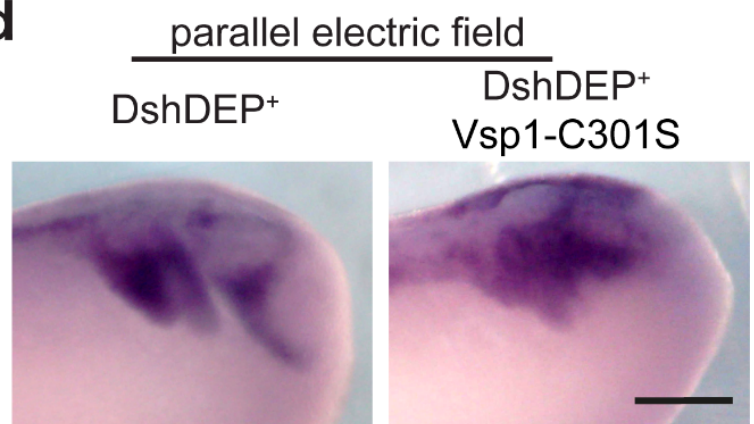
Fig. 4 | Application of parallel electric fields rescues PCP-dependent impaired collective cell migration in vivo. a, Scheme of $\mathrm{DshDEP}^{+}$targeted injection into the neural fold. $\mathbf{b}$, In situ hybridisations showing the effect of this injection in embryos in which no parallel electric fields (EFs) have been applied (lateral view of embryos hybridised with sox8, a neural crest migration marker). Scale bar, $200 \mu \mathrm{m}$. c, Scheme of the application of parallel EFs $\left(100 \mathrm{mV} \mathrm{mm} \mathrm{mm}^{-1}\right)$ in embryos with DshDEP ${ }^{+}$injected neural fold or in embryos with both DshDEP ${ }^{+}$-injected neural folds and Vsp1-C301S-injected neural crests. d, In situ hybridisations showing the effect of these treatments. Scale bar, $200 \mu \mathrm{m}$. e, Percentage of embryos displaying streams, column bars represent mean and error bars the standard deviation, Fisher's exact test (two-tailed): ${ }^{* * * *} p<$ 0.0001 for Control vs. DshDEP $; p=0.1176$ for Control vs. $\mathrm{DshDEP}^{+}+\mathrm{EFs}$; $* * p=0.008$ for $\mathrm{DshDEP}^{+}$vs. DshDEP ${ }^{+}+\mathrm{EFs} ; * * * * p<0.0001$ for Control vs. DshDEP ${ }^{+}+\mathrm{Vsp}^{*} 1-\mathrm{C} 301 \mathrm{~S}+\mathrm{EFs}$; ${ }^{* *} p=0.0058$ for $\mathrm{DshDEP}^{+}+\mathrm{EFs} \mathrm{vs} \mathrm{DshDEP}^{+}+\mathrm{Vsp} 1-\mathrm{C} 301 \mathrm{~S}+\mathrm{EFs}$. f, Neural crest streams normalised displacement, red lines represent median and error bars the interquartile ranges, Mann Whitney $U$-test, (two-tailed): ${ }^{* * *} p=0.0004$ for Control vs. $\operatorname{DshDEP}^{+} ; p=0.1098$ for Control vs. DshDEP + EFs; $* * * * p<0.0001$ for $\operatorname{DshDEP}^{+}$vs. $\operatorname{DshDEP}^{+}+\mathrm{EFs}^{* *} p=0.0023$

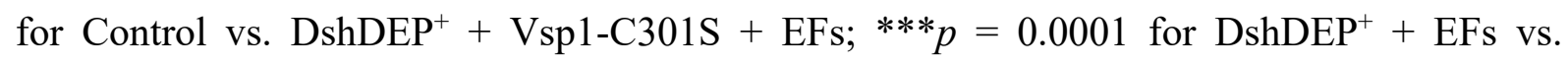
$\mathrm{DshDEP}^{+}+$Vsp1-C301S + EFs; $n_{\text {Control }}=43$ embryos, $n_{\mathrm{DshDEP}^{+}}=46$ embryos, $n_{\mathrm{DshDEP}^{+}+\mathrm{EFs}}=$ 22 embryos, $n_{\text {DshDEP }^{+}+\text {Vsp1-C301S }+ \text { EFs }}=20$ embryos. b,d, Representative examples from at least three independent experiments; $\mathrm{CI}=95 \%$. g, Schematic summarising the mechanism of neural crest collective electrotaxis discovered here: PCP-driven membrane stretch allow for ion mobilization in the neural fold. This, in addition to physiological inward currents in the flanking non-neural ectoderm, allow the establishment of a sustained electric field along the migratory path of neural crest cells (cyan). Then, the activity of Vsp1 is required in the neural crest to specifically transduce these electric stimuli into dCCM. 


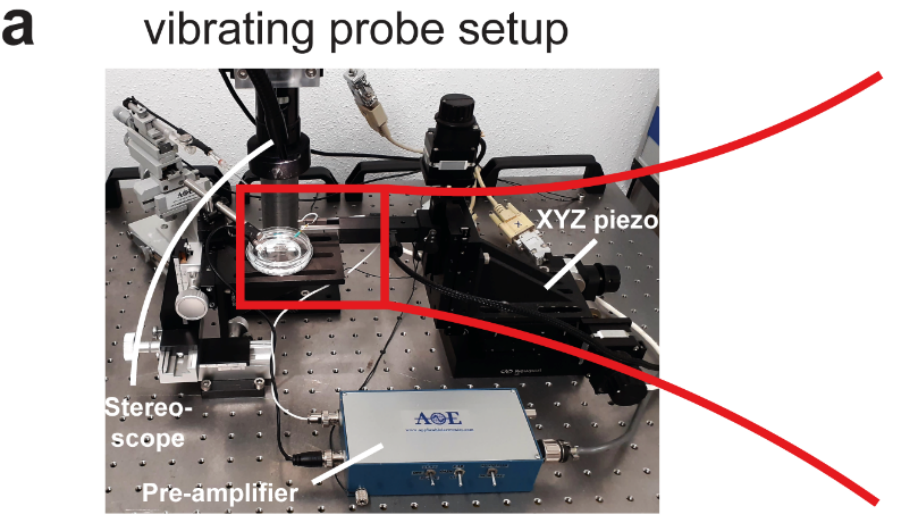

C
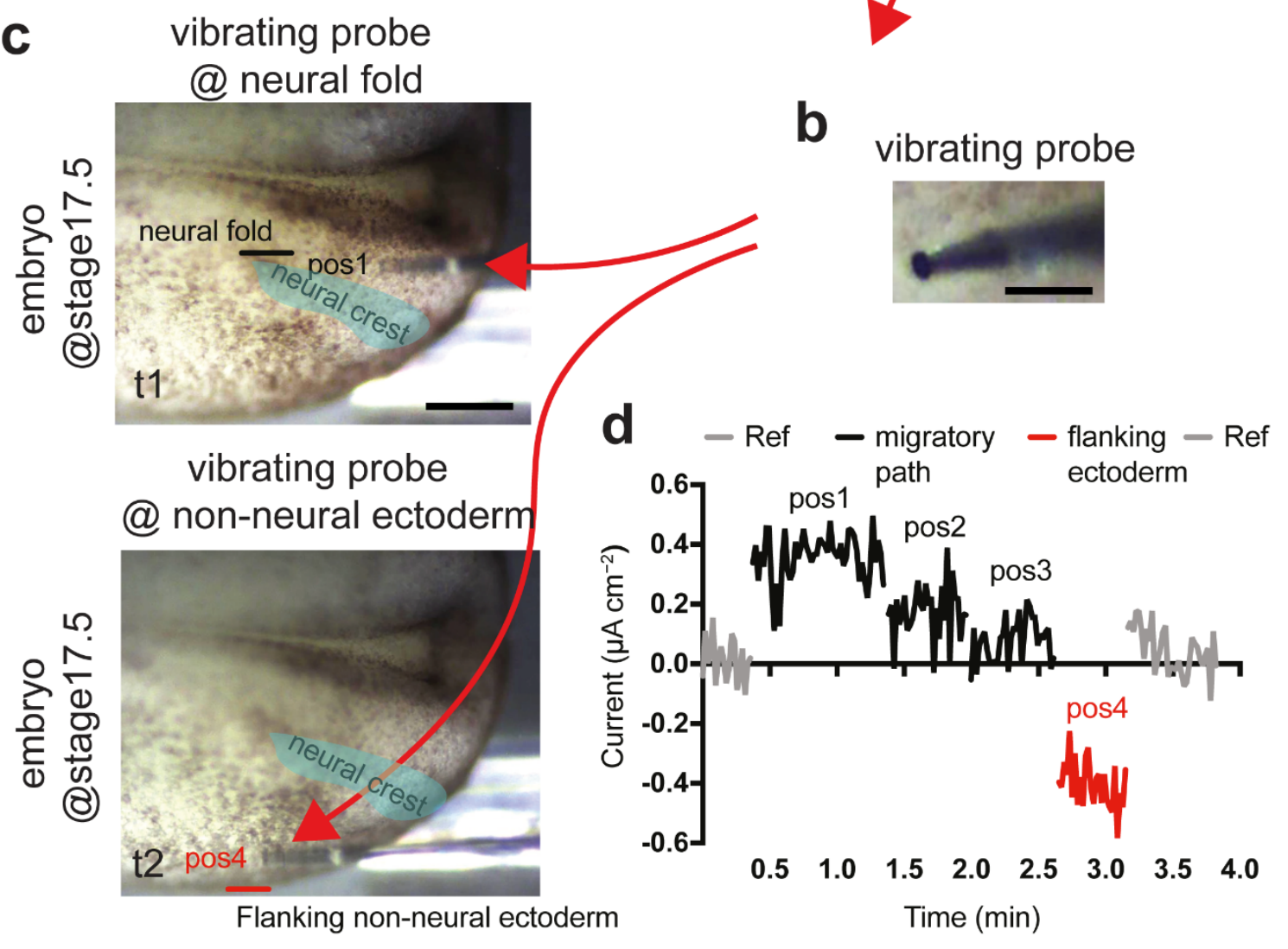

Supplementary Fig. 1 | Vibrating probe setup and measurements approach. a, Overview of the vibrating probe setup to measure electric current densities in vivo. Zoom in of the custommade measuring chamber. $\mathbf{b}$, Detail of the probe in static mode, note the platinum black ball ( $\sim 25 \mu \mathrm{m}$ diameter) electroplated to the tip. c, Representative photomicrographs acquired during a typical measurement; the probe in vibrating mode is indicated by a red arrow and shown in the neural fold (pos1) and then in the flanking non-neural ectoderm (pos4); other two records (pos2 and 3) were made between pos1 and 2, so the whole migratory path of neural crest cells was covered in each analysed embryo. Relative position of the neural crest is indicated in fading 
cyan. d, Representative result of the mediolateral records; pos1-pos4, positions along the migratory path, from neural fold to non-neural flanking ectoderm positions and Ref, reference (measurement obtained by holding the probe away from embryo). 


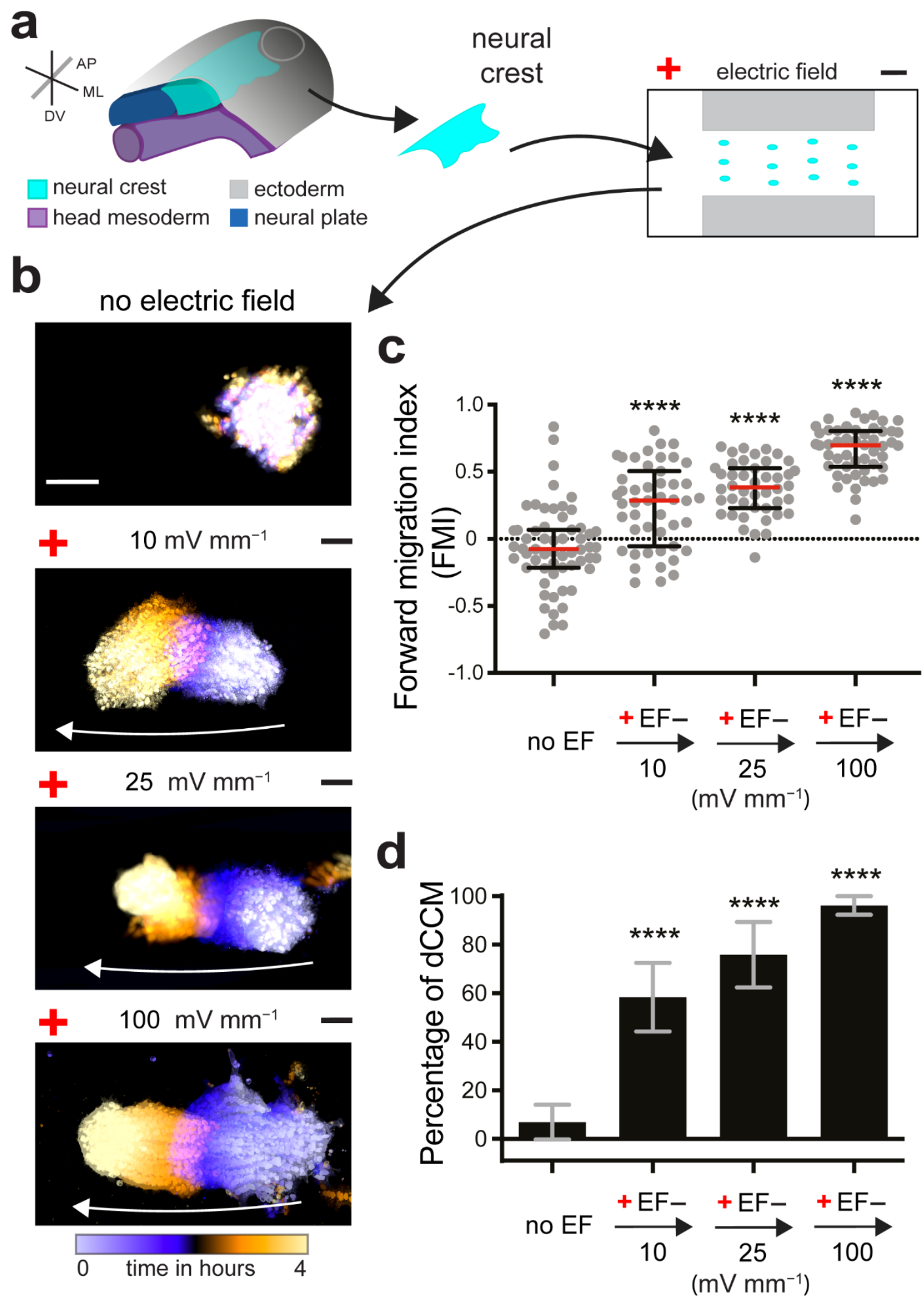


bioRxiv preprint doi: https://doi.org/10.1101/2021.10.11.463916; this version posted October 12,2021 . The copyright holder for this preprint (which was not certified by peer review) is the author/funder, who has granted bioRxiv a license to display the preprint in perpetuity. It is made available under aCC-BY-NC-ND 4.0 International license.

Supplementary Fig. 2 | Ex vivo electrotactic response of neural crest cells at the different magnitudes of electric fields recorded in vivo. a, Scheme of the electrotaxis assay. b, Time colour-coded trajectories of neural crest clusters, white arrows depict the cluster trajectories (see Supplementary Video 2). Scale bar, $100 \mu \mathrm{m}$. c, Forward migration index (FMI) quantifications, red lines represent median and error bars the interquartile ranges, One-way ANOVA with Dunnett's multiple comparisons test, ${ }^{* * * *} p<0.0001$ (two-tailed) for all electric fields (EFs) vs. No EFs, $n_{\mathrm{No} \text { EFs }}=59$ clusters, $n_{10} \mathrm{mV} \mathrm{mm}^{-1}=47$ clusters, $n_{25} \mathrm{mV} \mathrm{mm}^{-1}=44$ clusters, $n_{100 \mathrm{mV} \mathrm{mm}}{ }^{-1}=50$ clusters. d, Percentage of clusters displaying migration towards the anode; column bars represent mean and error bars the standard error, Fisher's exact test, ${ }^{* * * *} p<$ 0.0001 (two-tailed) for all EFs vs. No EFs, $n_{\mathrm{No} \text { EFs }}=59$ clusters, $n_{10} \mathrm{mV} \mathrm{mm}^{-1}=47$ clusters, $n_{25 \mathrm{mV}}$

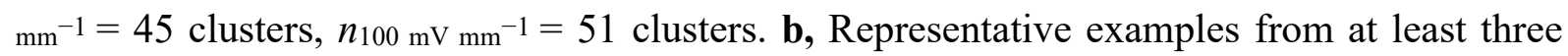
independent experiments; $\mathrm{CI}=95 \%$. 


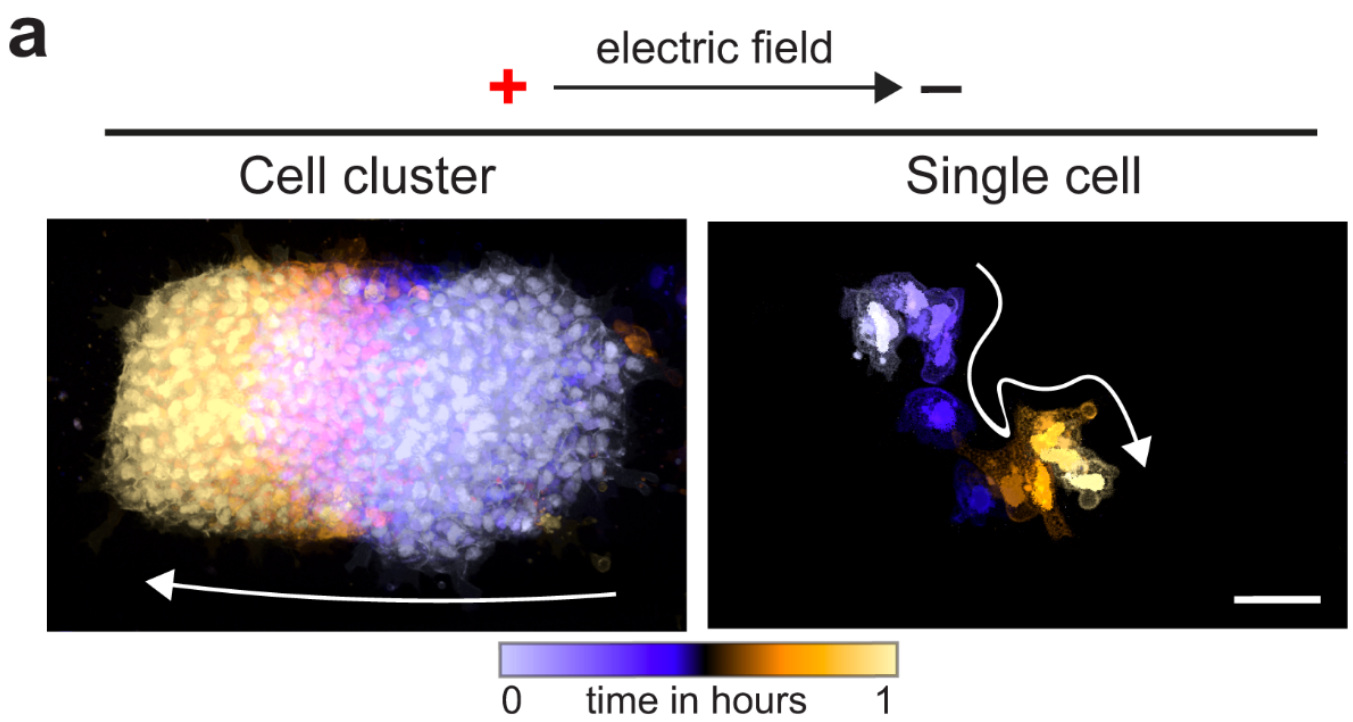

(2)

Cell cluster

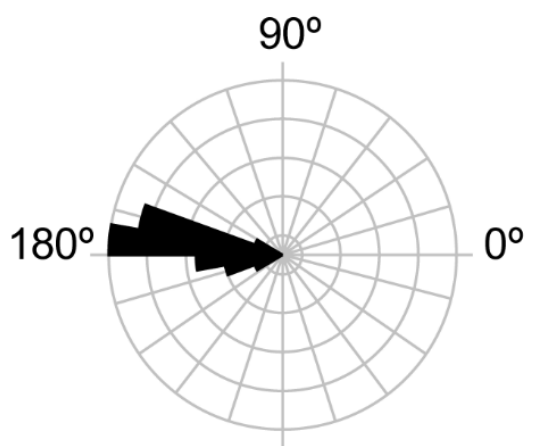

$270^{\circ}$
Single cell

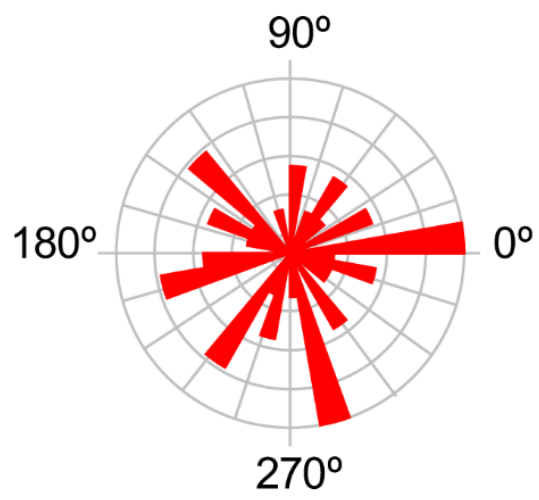

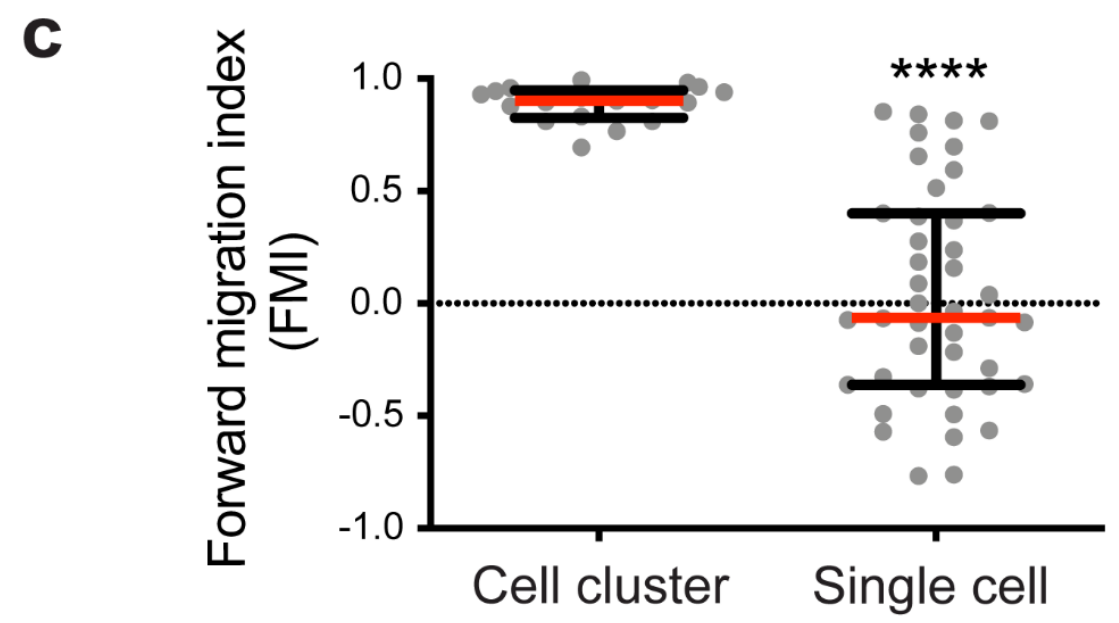

Supplementary Fig. 3 | Electrotaxis is an emergent property of cell clusters. a, Time colourcoded trajectories of neural crest clusters and isolated neural crest cells migrating in electric fields of $100 \mathrm{mV} \mathrm{mm}^{-1}$, white arrows depict the cluster or cell trajectories (see Supplementary 
Video 3). Scale bar, $100 \mu \mathrm{m}$. b, Rose plots showing the angle frequencies of neural crest migration in relation to the electric field vector (anode at $180^{\circ}$ ). c, Forward migration index (FMI) quantifications, red lines represent median and error bars the interquartile ranges, Student's $t$-test with Welch's correction, ${ }^{* * * *} p<0.0001$ (two-tailed), $n_{\text {Clusters }}=18, n_{\text {Single cells }}=$ 43. a, Representative examples from at least three independent experiments; $\mathrm{CI}=95 \%$. 
a

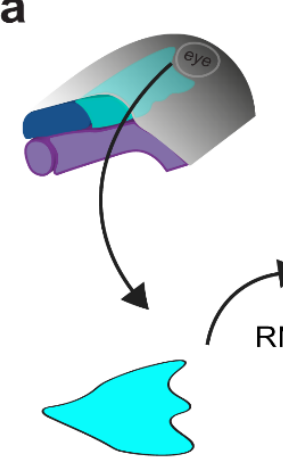

Neural Crest
$\mho_{D V}^{A P}$

RNA extraction

RNA-seq / bioinformatic analyses / validation

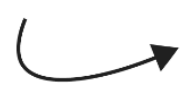

b

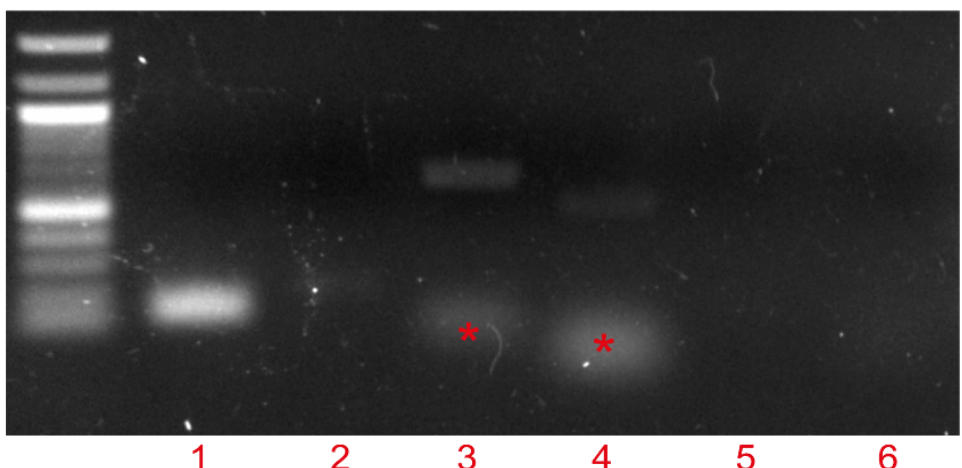

1 ef1 $\alpha(134 \mathrm{bp}) \quad 3$ sox8 (642 bp) 5 vsp2 (563 bp) * primer

2 krt12 (217 bp) 4 vsp1 (517 bp) 6 xbra (243 bp) dimer c

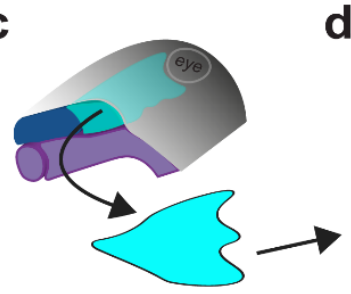

Neural Crest

e

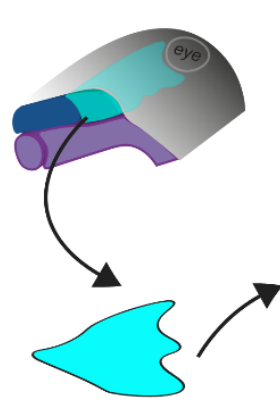

Neural Crest cell cluster d membrane

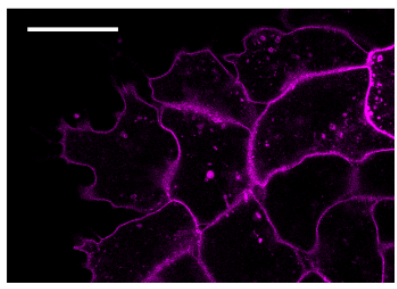

f membrane
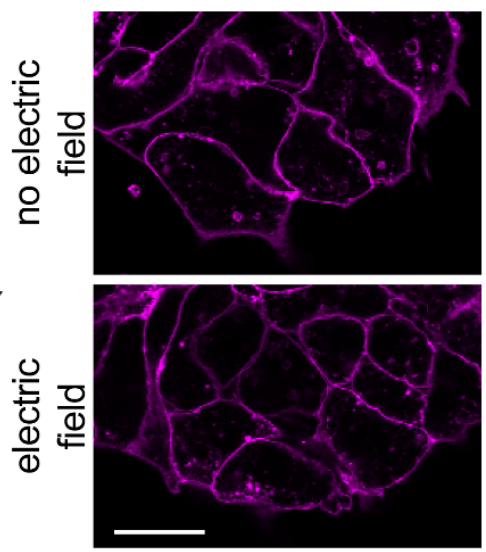

g

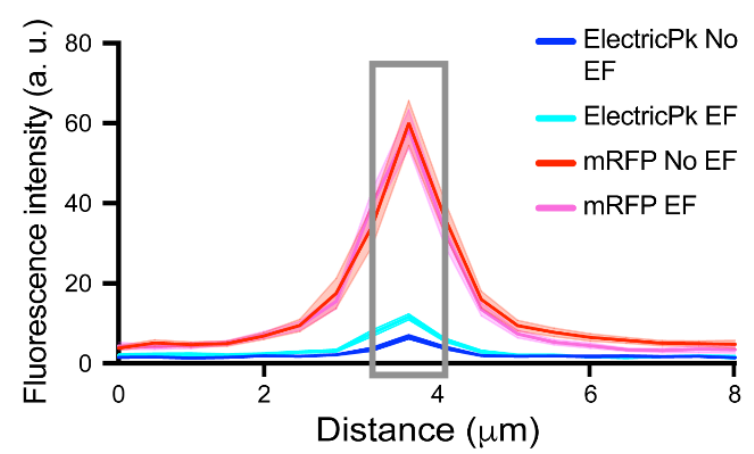

Vsp1-GFP

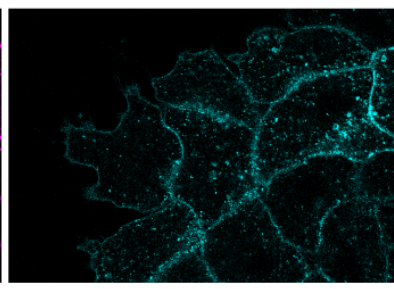

ElectricPK
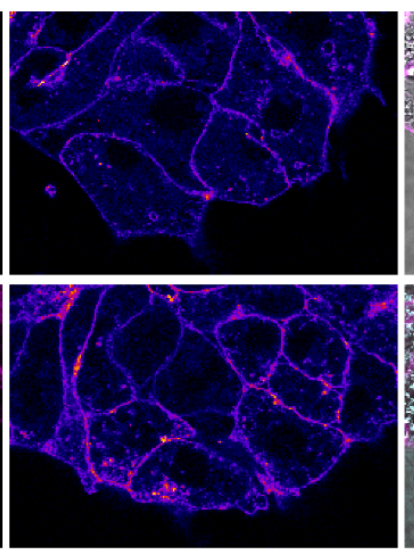

h merged

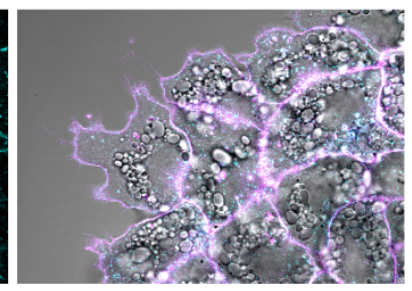

merged
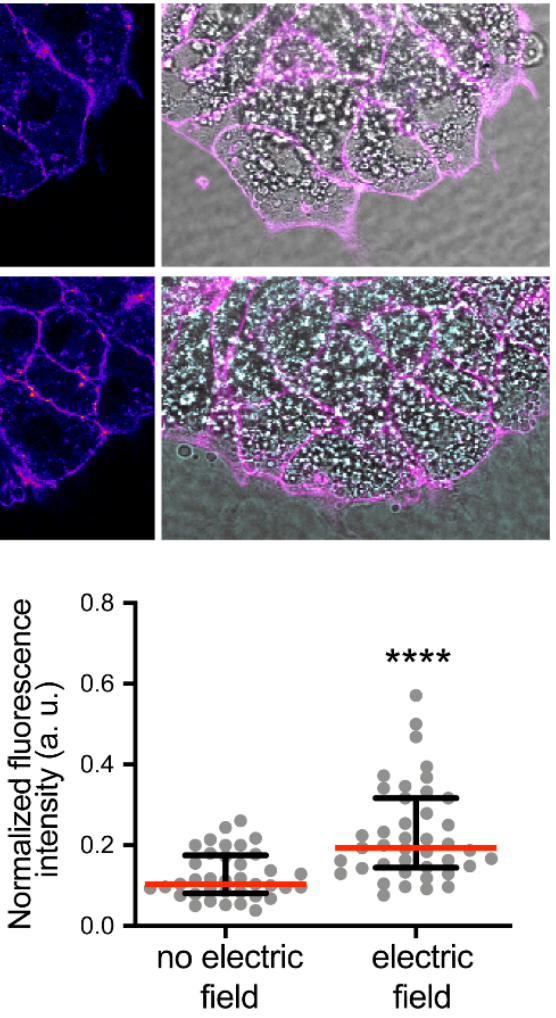

Supplementary Fig. 4 | Neural crest cells express the voltage-sensitive phosphatase, Vsp1.

a, Scheme and workflow of RNA extraction from neural crest explants dissected from premigratory embryos at st17-18 and processed for RNA-seq. b, $v s p 1$, but not $v s p 2$, is present in 
the neural crest cells. Purity of the explants further assessed by semiquantitative RT-PCR by confirming the presence of the neural crest marker (sox8) and the absence of ectodermal (krt12) and mesodermal ( $x b r a)$ markers (ruling out contamination with surrounding tissues). c, Scheme of dissection of the neural crest. d, Membrane localization of Vsp1 in a neural crest cluster. e, Scheme of dissection of the neural crest. f, Activity of Vsp1 in neural crest clusters in the absence or presence of electric fields (EFs) of $100 \mathrm{mV} \mathrm{mm}^{-1}$ for $1 \mathrm{~h}$ as reported by the ElectricPk-cpEGFP construct. ElectricPk-cpEGFP intensity is colour-coded in fire lookup table (hot colours represent higher intensity). g, Fluorescence intensity profile of lines overlaying membranes, solid lines represent mean and shades the standard error. $\mathbf{h}$, Maximum intensity normalized against the membrane RFP (grey rectangle in $\mathbf{g}$ ). Red lines represent median and error bars the interquartile ranges, Mann Whitney $U$-test, $* * * * p<0.0001$ (two-tailed), $n_{\mathrm{No} \text { EF }}=$ 37 cell membranes, $n_{\mathrm{EF}}=41$ cell membranes. Scale bars in $\mathbf{d}$ and f, $20 \mu \mathrm{m}$. b,d,f, Representative examples from at least three independent experiments; $\mathrm{CI}=95 \%$. 


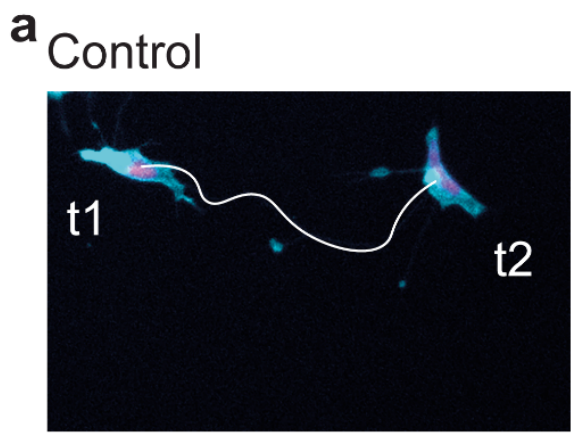

\section{Control}
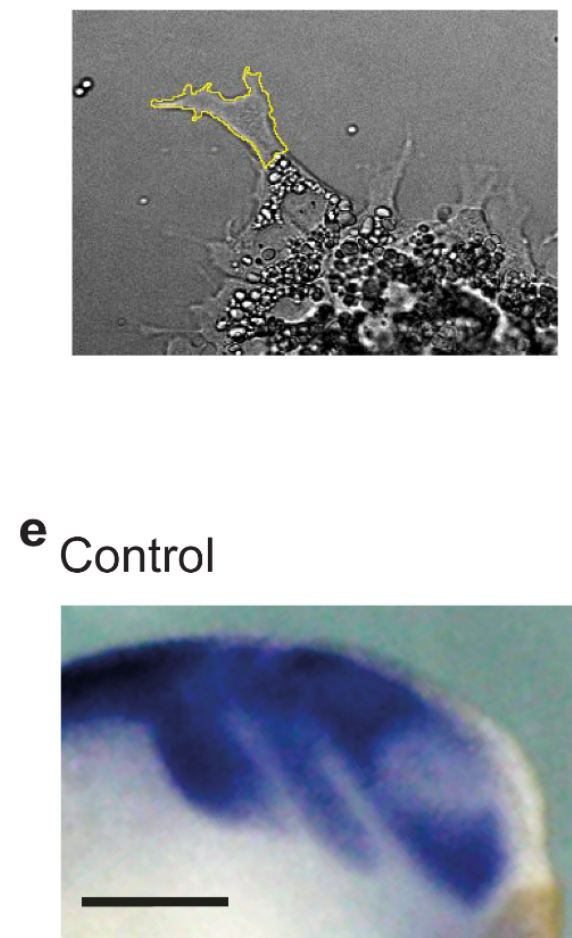

Vsp1-C301S

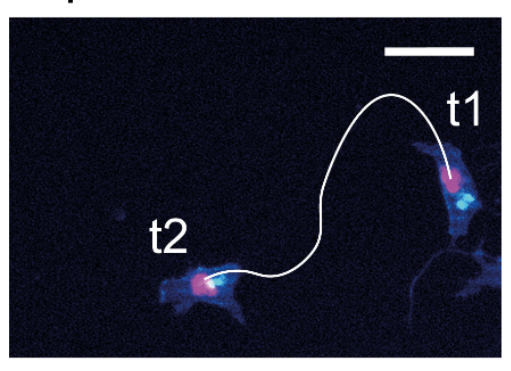

Vsp1-C301S

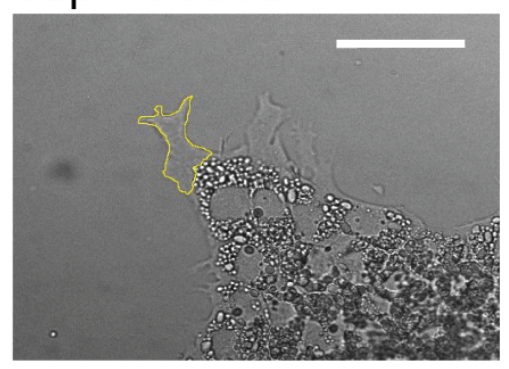

Vsp1-C301S

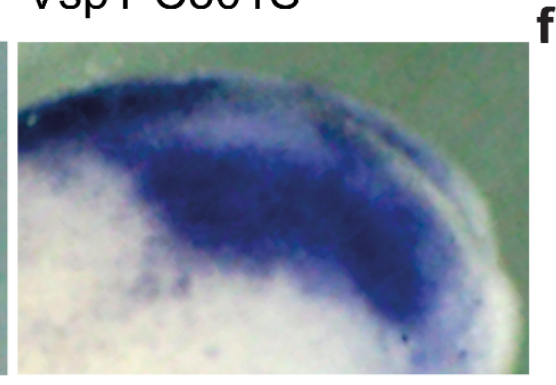

b
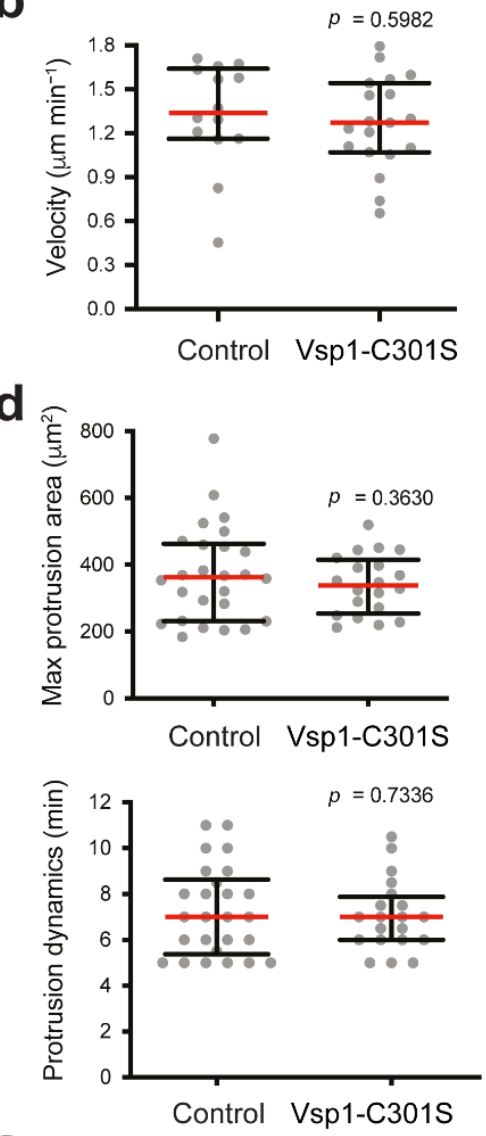

f

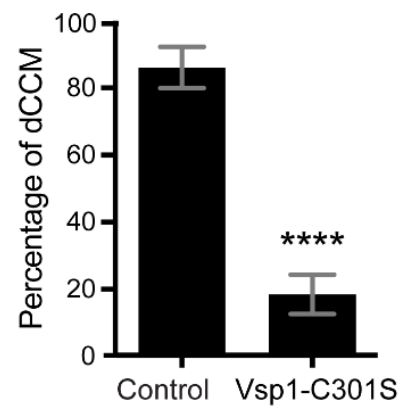

Supplementary Fig. 5 | Vsp1-C301S does not affect for neural crest cells motility ex vivo but it impairs stream formation in vivo. a, Overlay of isolated neural crest cells at t1 ( 0 min) and $\mathrm{t} 2(60 \mathrm{~min})$ of migration, solid white lines depict cell trajectories (see Supplementary Video 5). Scale bar, $30 \mu \mathrm{m}$. b, Quantification of single cells velocity, red lines represent median and error bars the interquartile ranges, $t$-test, $p=0.5982$ (two-tailed), $n_{\text {Control }}=14$ cells, $n$ Vsp1$\mathrm{C} 301 \mathrm{~S}=19$ cells. $\mathbf{c}$, Neural crest clusters with outlined protrusions (yellow solid lines). Scale bar $30 \mu \mathrm{m}$. Quantification of maximum protrusion area (d) and protrusion dynamics (e), red lines represent median and error bars the interquartile ranges, $t$-test (d) with Welch's correction (e), $p_{\text {Max Area }}=0.3630$ (two-tailed), $p_{\text {Dynamics }}=0.7336$ (two-tailed), $n_{\text {Control }}=26$ protrusions, $n_{\text {Vsp1- }}$ $\mathrm{C} 301 \mathrm{~S}=20$ protrusions. f,g, Vsp1-C301S impairs stream formation (a hallmark of dCCM) in 
vivo. f, In situ hybridisations showing lateral views of embryos hybridised with $c 3$ (a marker of neural crest migration). Scale bar, $200 \mu \mathrm{m}$. g, Percentage of embryos displaying stream formation, column bars represent mean and error bars the standard error, Fisher's exact test, $* * * * p<0.0001$ (two-tailed), $n_{\text {Control }}=25$ embryos, $n_{\mathrm{Vsp1-C} 301 \mathrm{~S}}=24$ embryos. a,c,f, Representative examples from at least three independent experiments; $\mathrm{CI}=95 \%$. 
a
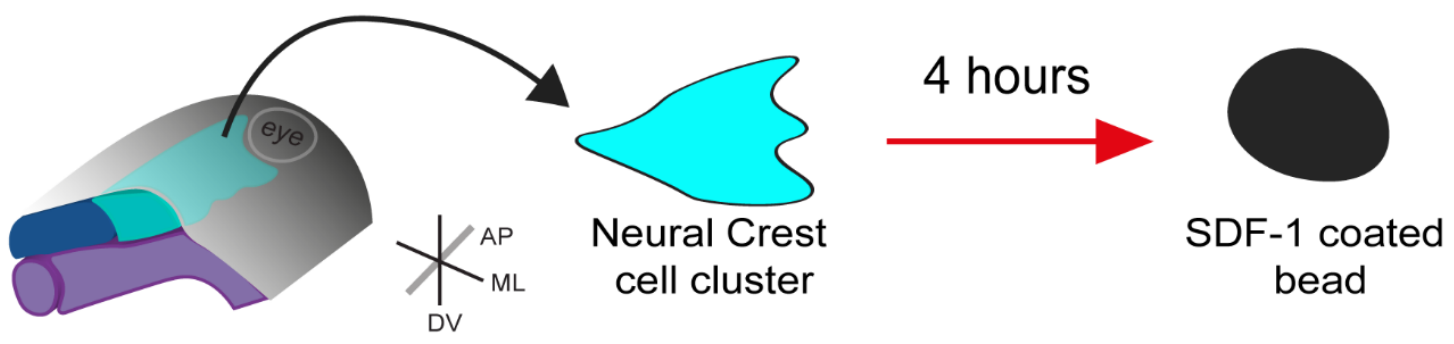

b

Control
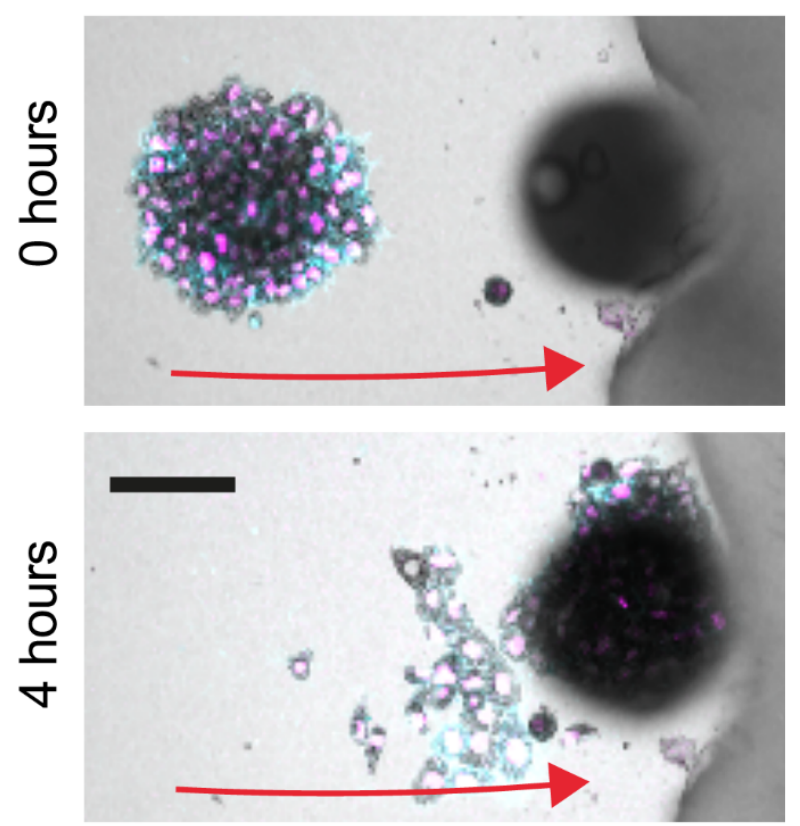

C

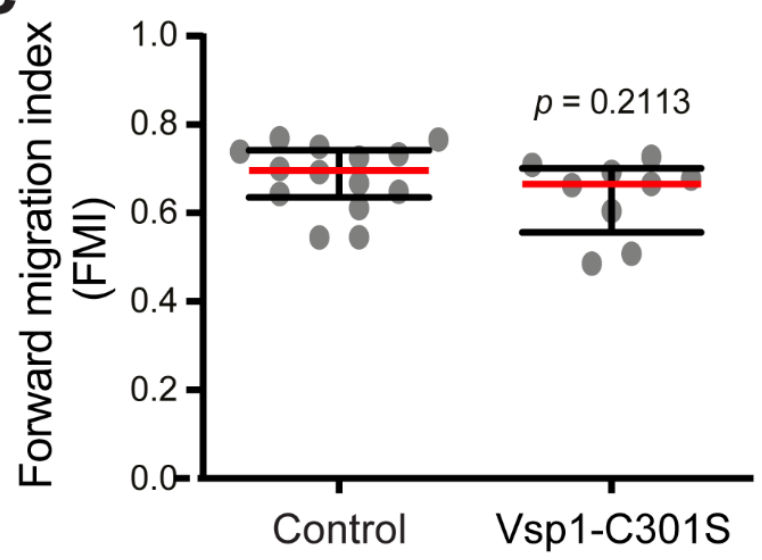

d

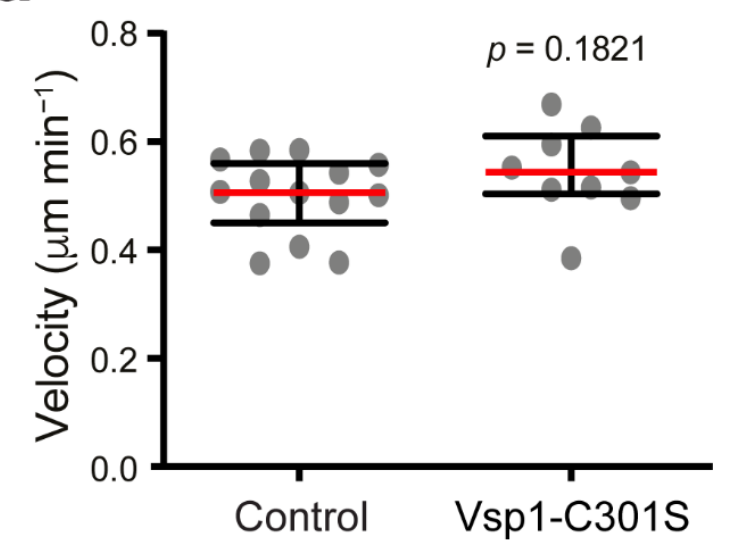

Supplementary Fig. 6 | Vsp1-C301S does not affect chemotaxis of neural crest cells ex vivo. a, Scheme of a typical chemotaxis assay. Neural crest clusters were exposed to a gradient of 
the chemoattractant SDF-1. b, Neural crest clusters migrating towards a SDF-1-coated bead, upper panels show clusters after 5 min of imaging and lower panels show clusters arriving to the SDF-1 bead after 4 hours of time-lapse (red arrows depict the direction of movement). Scale bar, $100 \mu \mathrm{m}$. Quantifications of forward migration index (FMI) (c) and velocity (d) of neural crest clusters, red lines represent median and error bars the interquartile range, Student's $t$-test, $p_{\text {FMI }}=0.2113$ (two-tailed), $p_{\text {Velocity }}=0.1821$ (two-tailed), $n_{\text {Control }}=14$ clusters, $n_{\text {Vsp1-C301S }}=9$ clusters. b, Representative examples from at least three independent experiments; $\mathrm{CI}=95 \%$. 
a

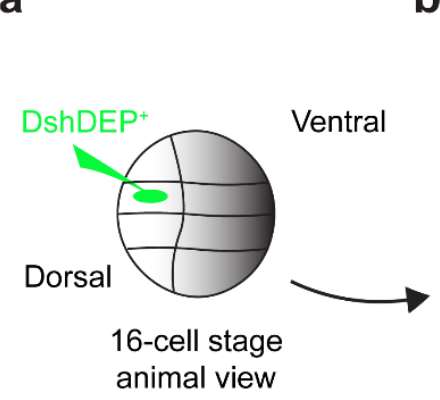

d

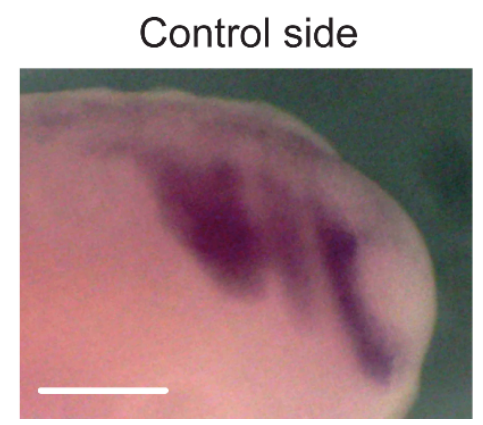

b
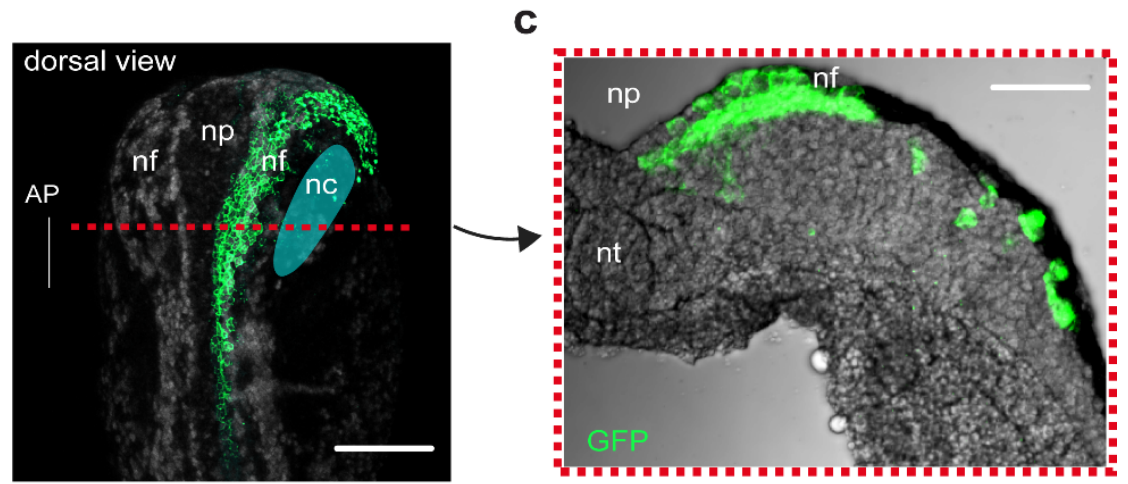

\section{Supplementary Fig. 7 | Targeted injection of DshDEP ${ }^{+}$in the neural fold affects neural} crest dCCM in a nonautonomous manner. a, Scheme of neural fold-targeted injection. b, Neural fold-tagged embryo at st17 (dorsal view), red dashed line shows the transverse plane of a cryosection presented in c. c, Cryosection showing the precise distribution of $\mathrm{DshDEP}^{+}$and the membrane GFP in the ectoderm at the level of the neural fold. Faded cyan ellipse, anatomical position of the neural crest (nc). nf, neural fold; $\mathrm{np}$, neural plate; nt, neural tube; AP, anteroposterior. d, In situ hybridisations showing lateral views of embryos hybridised with sox8 (a marker of neural crest migration). Scale bars, $200 \mu \mathrm{m}$. b-d, Representative examples from at least three independent experiments; $\mathrm{CI}=95 \%$. 
a

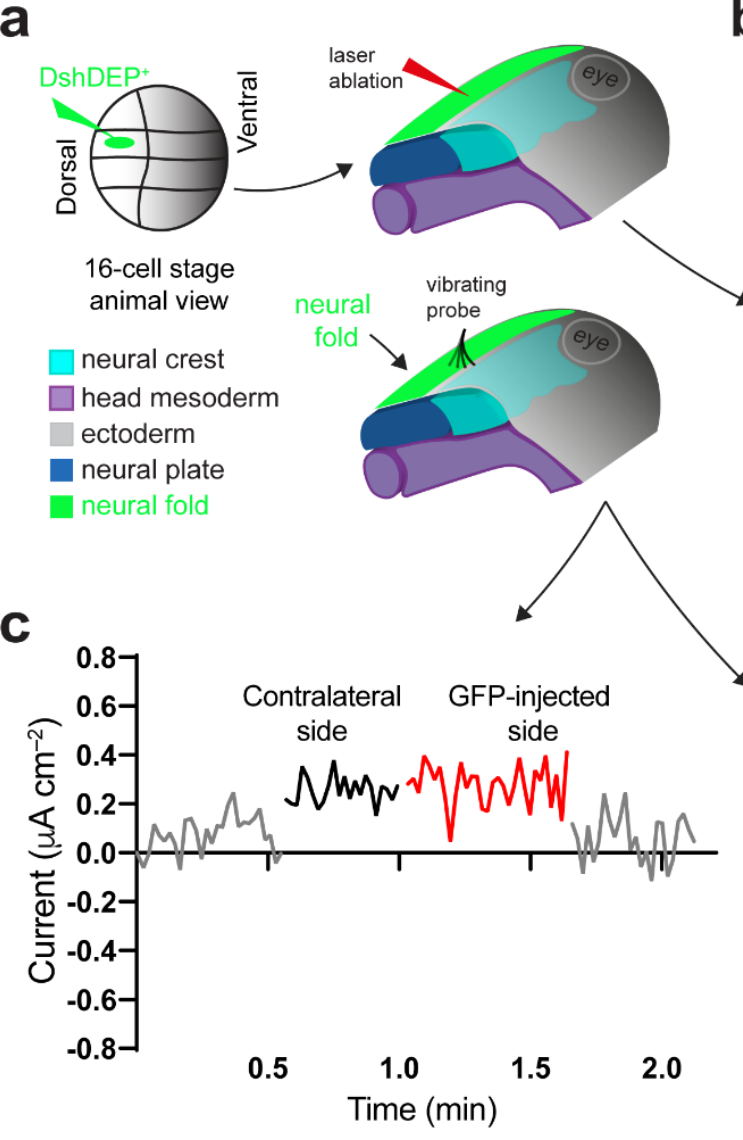

b
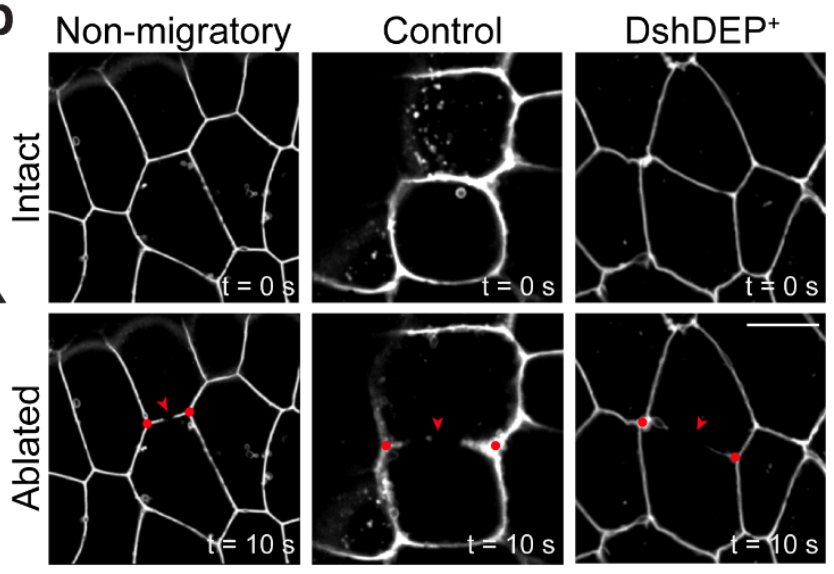

d

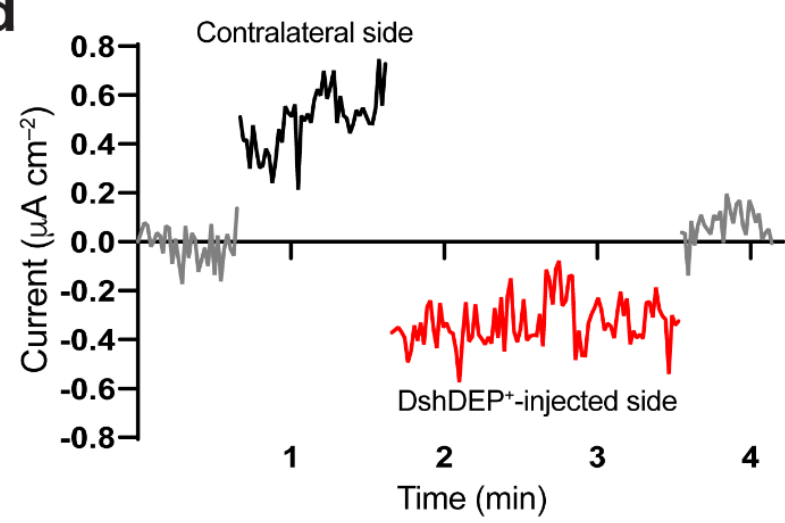

\section{Supplementary Fig. 8 | DshDEP ${ }^{+}$targeted injection in the neural fold reduces membrane}

tension and electric currents in vivo. a, Scheme of $\mathrm{DshDEP}^{+}$targeted injection into the neural fold for laser ablation and current measurements. b, Impact of stage and PCP inhibition in neural fold membrane recoil velocity as a function of stored tension (see Supplementary Video 9). Injection of GFP does not affect outward currents (c) but $\operatorname{DshDEP}^{+}$does (d). b, Representative examples from at least three independent experiments; CI $=95 \%$. 
bioRxiv preprint doi: https://doi.org/10.1101/2021.10.11.463916; this version posted October 12, 2021. The copyright holder for this preprint (which was not certified by peer review) is the author/funder, who has granted bioRxiv a license to display the preprint in perpetuity. It is made available under aCC-BY-NC-ND 4.0 International license.

Supplementary Table 1 | vsp1 (tpte2.L) is present in neural crest cells RNA-seq libraries. The list provides the ID and names for the most represented ion channels found in our RNA library. These channels were selected as they are members of the voltage-gated ion channel families (https://www.genenames.org). The voltage-sensitive phosphatase $v s p 1$ (tpte2.L) emerged from this list and its paralogous vsp2 (tpte2.S) was not detected, as previously described for these stages ${ }^{62}$. Genes are shown in the same order as in the normalized TMM (Trimmed Mean of Mvalues) of the RNA-seq data analysis (Methods).

\begin{tabular}{ll}
\hline Gene ID & Gene name \\
\hline gene8850 & cacna1a.S \\
gene48032 & cacna2d3.L \\
gene16978 & cacna1c.L \\
gene16045 & hvcn1.L \\
xgene9796 & hvcn1.S \\
gene7902 & kcna2.L \\
gene10259 & tpte2.L \\
gene1014 & cacna1s.S \\
gene48712 & cacna2d1.L \\
gene7350 & kcna4.L \\
gene11153 & kcnq1.L \\
gene7292 & cacna2d2.L \\
gene7025 & scn5a.S \\
gene20266 & scn3b.L \\
gene15291 & cacnb1.L \\
gene12372 & cacng5.L \\
gene49655 & cacng4.L \\
gene9207 & cacnb4.L \\
gene38977 & cacng4.S \\
\hline gene9633 & cacnb4.S \\
\hline
\end{tabular}

$v s p 1$ (tpte2.L) gene highlighted in grey. 
bioRxiv preprint doi: https://doi.org/10.1101/2021.10.11.463916; this version posted October 12, 2021. The copyright holder for this preprint (which was not certified by peer review) is the author/funder, who has granted bioRxiv a license to display the preprint in perpetuity. It is made available under aCC-BY-NC-ND 4.0 International license.

Supplementary Table $2 \mid$ VSP (TPTE2) expression in cell lines that have been reported to electrotax. In silico analysis reveals expression of orthologous VSP in wound healing and cancer cells that are well known to follow the electric fields in vitro.

\begin{tabular}{|c|c|c|c|}
\hline Cell type & $\begin{array}{l}\text { Primary culture } \\
\text { or cell line }\end{array}$ & VSP (TPTE2) expression & $\begin{array}{l}\text { Electrotactic } \\
\text { response }\end{array}$ \\
\hline $\begin{array}{l}\text { Mammary } \\
\text { epithelium }\end{array}$ & MCF-10A & Lusche et al., $2018^{63}$ & $\begin{array}{l}\text { Lalli and } \\
\text { Asthagiri, } \\
2015^{64}\end{array}$ \\
\hline Fibroblasts & NIH 3T3* & $\begin{array}{l}\text { BioGPS (Mouse Cell Type and } \\
\text { Tissue Gene Expression Profiles) }{ }^{65-68}\end{array}$ & $\begin{array}{l}\text { Huang et al., } \\
2013^{69}\end{array}$ \\
\hline \multirow[t]{2}{*}{$\begin{array}{l}\text { Human breast } \\
\text { cancer }\end{array}$} & $\mathrm{MCF}-7^{*}$ & $\begin{array}{l}\text { Expression Atlas } \\
(\text { www.ebi.ac.uk/gxa })^{70}\end{array}$ & $\begin{array}{l}\text { Pu et al., } \\
2007^{71}\end{array}$ \\
\hline & MDA-MB-231* & $\begin{array}{l}\text { Harmonizome (Cell Line Gene } \\
\text { Expression Profiles) } \\
67,72\end{array}$ & $\begin{array}{l}\text { Wu et al., } \\
2013^{73}\end{array}$ \\
\hline Macrophages & Primary culture* & $\begin{array}{l}\text { BioGPS (Mouse Cell Type and Tissue } \\
\text { Gene Expression Profiles) }\end{array}$ & $\begin{array}{l}\text { Hoare et al., } \\
2014^{74}\end{array}$ \\
\hline
\end{tabular}

*VSP (TPTE2) expression reported in publicly available databases. 


\section{Supplementary Video Legends}

\section{Supplementary Video 1 | Neural crest cells migrate towards the anode of electric fields $e x$} vivo. Representative time-lapses of neural crest clusters in the absence (upper panel) or presence (lower panel) of electric fields (EFs) for $8 \mathrm{~h}$. The lower panel cluster was exposed to EFs of $100 \mathrm{mV} \mathrm{mm}^{-1}$ for $4 \mathrm{~h}$, with polarity reversal for another $4 \mathrm{~h}$. Time-lapse setting was 1 picture every $5 \mathrm{~min}$; 96 frames are shown at 7 frames per second. Note the cluster spreading and dispersing in the absence of EFs and the consistent anodal migration in the presence of EFs. Neural crest cells tagged with nuclear RFP (magenta) and membrane GFP (cyan) constructs. Scale bar, $100 \mu \mathrm{m}$. Related to Fig. 1.

\section{Supplementary Video 2 | Neural crest cells electrotaxis ex vivo under a range of electric}

fields recorded in vivo. Representative time-lapses of neural crest clusters exposed to electric fields of 10, 25 and $100 \mathrm{mV} \mathrm{mm}^{-1}$. Time-lapse setting was 1 picture every $5 \mathrm{~min} ; 48$ frames are shown at 7 frames per second. Note the anodal migration. Neural crest cells tagged with nuclear RFP (magenta) and membrane GFP (cyan) constructs. Scale bar, $100 \mu \mathrm{m}$. Related to Supplementary Fig. 2.

\section{Supplementary Video 3 | Neural crest cells electrotact as a collective but not as single cells} ex vivo. Representative time-lapses of a neural crest cluster and an isolated neural crest cell exposed to electric fields of $100 \mathrm{mV} \mathrm{mm}^{-1}$. Time-lapse setting was 1 picture every $5 \mathrm{~min} ; 12$ frames are shown at 7 frames per second. Note the anodal migration of the cluster and the random migration of the cell. Neural crest cells tagged with nuclear RFP (magenta) and membrane GFP (cyan) constructs. Scale bar, $50 \mu \mathrm{m}$. Related to Supplementary Fig. 3.

\section{Supplementary Video 4 | Vsp1-C301S does not affect neural crest cells motility ex vivo.} Representative time-lapses showing the migration of isolated control or Vsp1-C301S-injected cells. Time-lapse setting was 1 picture every $5 \mathrm{~min} ; 12$ frames are shown at 7 frames per second. Note the similar motility of both conditions. Neural crest cells tagged with nuclear RFP (magenta) and membrane GFP (cyan) constructs. Scale bar, $30 \mu \mathrm{m}$. Related to Supplementary Fig. 5. 
Supplementary Video 5 | Vsp1-C301S impairs neural crest electrotaxis ex vivo. Representative time-lapses of control and Vsp1-C301S-injected neural crest clusters exposed to electric fields of $100 \mathrm{mV} \mathrm{mm}^{-1}$. Time-lapse setting was 1 picture every $5 \mathrm{~min} ; 36$ frames are shown at 7 frames per second. Note the anodal migration of the control cluster and the random migration of the Vsp1-C301S cluster. Neural crest cells tagged with nuclear RFP (magenta) and membrane GFP (cyan) constructs. Scale bar, $100 \mu \mathrm{m}$. Related to Fig. 2.

\section{Supplementary Video 6 | Vsp1-R152Q allows for neural crest electrotaxis under} suboptimal electric fields ex vivo. Representative time-lapses of control and Vsp1-R152QGFP-injected neural crest clusters exposed to suboptimal electric fields of $5 \mathrm{mV} \mathrm{mm}{ }^{-1}$. Timelapse setting was 1 picture every 5 min; 72 frames are shown at 7 frames per second. Note the random migration of the control cluster and the anodal migration of the Vsp1-R152Q cluster. Neural crest cells tagged with nuclear RFP construct in cyan; fusion GFP of the Vsp1-R152QGFP construct in green. Scale bar, $100 \mu \mathrm{m}$. Related to Fig. 2.

\section{Supplementary Video 7 | Vsp1-C301S affects neural crest directionality in vivo.} Representative time-lapses showing lateral views of wild-type embryos in which control or Vsp1-C301S-injected neural crest explants were grafted. Time-lapse setting was 1 picture every 5 min; 72 frames are shown at 7 frames per second. Note the persistent migration of control neural crests in contrast to the random migration of Vsp1-C301S clusters. Neural crest cells tagged with nuclear RFP construct in cyan. Embryo and eye (ellipse) outlined in solid white lines. Scale bar, $200 \mu \mathrm{m}$. Related to Fig. 2 and Supplementary Fig. 5.

\section{Supplementary Video 8 | Vsp1-C301S does not alter neural crest chemotaxis towards}

SDF-1 ex vivo. Representative time-lapses of control and Vsp1-C301S-injected neural crest clusters exposed to SDF-1-coated beads. Time-lapse setting was 1 picture every 5 min; 48 frames are shown at 7 frames per second. Note the similar directional migration (towards the bead) of both conditions. Neural crest cells tagged with nuclear RFP (magenta) and membrane GFP (cyan) constructs. Scale bar, $100 \mu \mathrm{m}$. Related to Supplementary Fig. 6. 


\section{Supplementary Video 9 | Non-migratory and DshDEP ${ }^{+}$-injected neural folds reduce}

membrane tension in vivo. Representative time-lapses of laser ablations (solid red line) in the membranes of non-migratory (st13), control (pre-migratory st17) and $\mathrm{DshDEP}^{+}$(pre-migratory st17) injected neural folds. Time-lapse setting was 1 picture every 2 seconds; 6 frames are shown at 4 frames per second. Note that the membrane junction recoil in control neural fold is higher than in non-migratory and $\mathrm{DshDEP}^{+}$neural folds. Neural fold cells tagged with membrane GFP construct in grey. Scale bar, $15 \mu \mathrm{m}$. Related to Fig. 3 and Supplementary Fig. 8.

\section{Supplementary Video 10 | PCP in the neural fold controls the directionality of neural crest}

cells in vivo. Representative time-lapses showing lateral views of embryos in which wild-type neural crest explants (nuclear RFP in cyan) were grafted into control embryos (injected with membrane GFP in the neural fold, shown in grey) or into embryos in which $\mathrm{DshDEP}^{+}$injection was targeted to the neural fold (in grey). Time-lapse setting was 1 picture every $5 \mathrm{~min}$; 60 frames are shown at 7 frames per second. Note that the wild-type neural crest directionally migrates in GFP but not in DshDEP ${ }^{+}$hosts with targeted neural folds. Scale bar, $200 \mu \mathrm{m}$. Related to Fig. 3. 


\section{References}

62. Ratzan, W., Rayaprolu, V., Killian, S. E., Bradley, R. \& Id, S. C. K. The voltage sensing phosphatase (VSP) localizes to the apical membrane of kidney tubule epithelial cells. PLoS One 14, 121 (2019).

63. Lusche, D. F. et al. Overexpressing TPTE2 (TPIP), a homolog of the human tumor suppressor gene PTEN, rescues the abnormal phenotype of the PTEN-/- mutant. Oncotarget 9, 21100-21121 (2018).

64. Lalli, M. L. \& Asthagiri, A. R. Collective Migration Exhibits Greater Sensitivity But Slower Dynamics of Alignment to Applied Electric Fields. Cell. Mol. Bioeng. 8, 247-257 (2015).

65. Su, A. I. et al. A gene atlas of the mouse and human protein-encoding transcriptomes. Proc. Natl. Acad. Sci. U. S. A. 101, 6062-6067 (2004).

66. Su, A. I. et al. Large-scale analysis of the human and mouse transcriptomes. Proc. Natl. Acad. Sci. U. S. A. 99, 4465-4470 (2002).

67. Rouillard, A. D. et al. The harmonizome: a collection of processed datasets gathered to serve and mine knowledge about genes and proteins. Database (Oxford). 2016, (2016).

68. Wu, C., MacLeod, I. \& Su, A. I. BioGPS and MyGene.info: Organizing online, gene-centric information. Nucleic Acids Res. 41, D561-D565 (2013).

69. Huang, Y. J., Samorajski, J., Kreimer, R. \& Searson, P. C. The Influence of Electric Field and Confinement on Cell Motility. PLoS One 8, 59447 (2013).

70. Papatheodorou, I. et al. Expression Atlas update: From tissues to single cells. Nucleic Acids Res. 48, D77-D83 (2020).

71. $\mathrm{Pu}$, J. et al. EGF receptor signalling is essential for electric-field-directed migration of breast cancer cells. J. Cell Sci. 120, 3395-3403 (2007).

72. Heiser, L. M. et al. Subtype and pathway specific responses to anticancer compounds in breast cancer. Proc. Natl. Acad. Sci. U. S. A. 109, 2724-2729 (2012).

73. Wu, D., Ma, X. \& Lin, F. DC Electric Fields Direct Breast Cancer Cell Migration, Induce EGFR Polarization, and Increase the Intracellular Level of Calcium Ions. Cell Biochem. Biophys. 67, 1115-1125 (2013).

74. Hoare, J. I., Rajnicek, A. M., McCaig, C. D., Barker, R. N. \& Wilson, H. M. Electric fields are novel determinants of human macrophage functions. J. Leukoc. Biol. 99, 1141-1151 (2016). 